\title{
SIMULATED LSST SURVEY OF RR LYRAE STARS THROUGHOUT THE LOCAL GROUP
}

\author{
Hakeem M. Oluseyi ${ }^{1}$, Andrew C. Becker ${ }^{2}$, Christopher Culliton ${ }^{1}$, Muhammad Furqan $^{1}$, Keri L. Hoadley $^{1}$, \\ Paul Regencia $^{1}$, Akeem J. Wells ${ }^{1}$, Željko Ivezic̀ ${ }^{2}$, R. Lynne Jones $^{2}$, K. Simon Krughoff $^{2}$, Branimir Sesar $^{3}$, \\ SuZANne JaCOBY ${ }^{4}$, AND IDATONYE J. Allison ${ }^{5}$ \\ ${ }^{1}$ Department of Physics and Space Sciences, Florida Institute of Technology, Melbourne, FL 32901, USA \\ ${ }^{2}$ Astronomy Department, University of Washington, Seattle, WA 98195, USA \\ ${ }^{3}$ Division of Physics, Mathematics and Astronomy, California Institute of Technology, Pasadena, CA 91125, USA \\ ${ }^{4}$ LSST Corporation, Tucson, AZ 85721, USA \\ ${ }^{5}$ Department of Physics, Alabama Agricultural and Mechanical University, Normal, AL 35762, USA \\ Received 2011 March 27; accepted 2012 April 13; published 2012 June 7
}

\begin{abstract}
We report on a study to determine the efficiency of the Large Synoptic Survey Telescope (LSST) to recover the periods, brightnesses, and shapes of RR Lyrae stars' light curves in the volume extending to heliocentric distances of $1.5 \mathrm{Mpc}$. We place the smoothed light curves of 30 type $a b$ and 10 type $c$ RR Lyrae stars in 1007 fields across the sky, each of which represents a different realization of the LSST sampling cadences, and that sample five particular observing modes. A light curve simulation tool was used to sample the idealized RR Lyrae stars' light curves, returning each as it would have been observed by LSST, including realistic photometric scatter, limiting magnitudes, and telescope downtime. We report here the period, brightness, and light curve shape recovery as a function of apparent magnitude and for survey lengths varying from 1 to 10 years. We find that 10 years of LSST data are sufficient to recover the pulsation periods with a fractional precision of $\sim 10^{-5}$ for $\geqslant 90 \%$ of $a b$ stars within $\approx 360 \mathrm{kpc}$ of the Sun in Universal Cadence fields and out to $\approx 760 \mathrm{kpc}$ for Deep Drilling fields. The $50 \%$ completeness level extends to $\approx 600 \mathrm{kpc}$ and $\approx 1.0 \mathrm{Mpc}$ for the same fields, respectively. For virtually all stars that had their periods recovered, their light curve shape parameter $\phi_{31}$ was recovered with sufficient precision to also recover photometric metallicities to within 0.14 dex (the systematic error in the photometric relations). With RR Lyrae stars' periods and metallicities well measured to these distances, LSST will be able to search for halo streams and dwarf satellite galaxies over half of the Local Group, informing galaxy formation models and providing essential data for mapping the Galactic potential. This study also informs the LSST science operations plan for optimizing observing strategies to achieve particular science goals. We additionally present a new $[\mathrm{Fe} / \mathrm{H}]-\phi_{31}$ photometric relation in the $r$ band and a new and generally useful metric for defining period recovery for time domain surveys.
\end{abstract}

Key words: Galaxy: halo - Galaxy: structure - methods: data analysis - stars: variables: RR Lyrae

Online-only material: color figures

\section{INTRODUCTION}

Understanding the details of galaxy formation and evolution are major objectives of modern astrophysics. Studies of the structure and dynamics of our Milky Way and nearby Local Group galaxies in particular advance a pathway to understanding the details of galaxy evolution in general. The space distributions (Ivezić et al. 2000, 2005; Sesar et al. 2007; Keller et al. 2008; Watkins et al. 2009; Sesar et al. 2010a), kinematics (Helmi \& White 1999; Kleyna et al. 2001, 2002, 2003, 2004), ages (Dolphin et al. 2001, 2004; Dolphin 2002a, 2002b), and chemistries of stellar overdensities (Prantzos 2008; Koch et al. 2009; Aoki et al. 2009; Koch \& Côté 2010) in galactic halos provide useful constraints on models of galaxy structure and evolution (Bullock \& Johnston 2005; Robertson et al. 2005; Johnston et al. 2008; De Lucia \& Helmi 2008; Frebel et al. 2008). Studies of these sorts have revealed ample evidence of hierarchical Galactic assembly as proposed by Searle \& Zinn (1978) including discoveries of accreted dwarf satellite galaxies, tidal streams of satellite galaxies and globular clusters, and stripped remnants of accreted satellites and globular clusters (Ibata et al. 1994, 1995, 2001a, 2001b, 2002; Newberg et al. 2002, 2003; Yanny et al. 2003; Odenkirchen et al. 2003;
Martin et al. 2004; Rocha-Pinto et al. 2004; Belokurov et al. 2006a, 2006b, 2006c, 2007a, 2007b, 2007c, 2008, 2009, 2010; Grillmair \& Johnson 2006; Grillmair \& Dionatos 2006a, 2006b; Grillmair 2006a, 2006b, 2009; Bell et al. 2008; Newberg et al. 2009; Watkins et al. 2009; Mackey et al. 2010a). Studies of M31's halo have also revealed an extended halo containing evidence of satellite accretions and tidal streams illustrating both similarities and differences with the Milky Way's halo (Durrell et al. 2001; Majewski et al. 2004, 2007; Brook et al. 2004; Ibata et al. 2004, 2005, 2007; Font et al. 2008; Dolphin et al. 2004; Mouhcine et al. 2005; Kalirai et al. 2006; Font et al. 2008; Kirby et al. 2009; Alves-Brito et al. 2009; Richardson et al. 2009; Tanaka et al. 2010; Mackey et al. 2010a, 2010b; Koch \& Rich 2010). These near-field cosmology studies of the distributions of individual stars in spatial-kinematic-metallicity-age space are invaluable for creating general models of galaxy formation and for establishing $\Lambda$-CDM as the dominant cosmological model (Seljak et al. 2006; Spergel et al. 2007; Read et al. 2008).

RR Lyrae variable stars are an exceptionally useful tracer population of Galactic structure. Their utility derives from simple relations correlating observable parameters, such as period, amplitude of pulsation, and metallicity, with evolutionary parameters such as luminosity (Marconi et al. 2006). Detections 
of overdensities of RR Lyrae stars have been successfully used to identify substructure within our own Galactic halo (Ivezić et al. 2000, 2005; Sesar et al. 2007, 2010a; Keller et al. 2008; Watkins et al. 2009). Metallicity estimates of detected RR Lyrae stars additionally constrain Galaxy accretion models that predict a difference in chemical composition between the inner (old accretion) and outer (recent accretion) halo (Bullock \& Johnston 2005; Johnston et al. 2008; Szczygieł et al. 2009). However, current RR Lyrae observations have been over a limited area, providing only volumetric slices through any extant Galactic halo substructure.

The complex and overlapping morphologies of accreted substructures seen in galaxy accretion simulations (Helmi et al. 1999; Johnston et al. 2008; Cooper et al. 2010) require fullsky surveys to trace and disentangle the observed halo streams. Overdensities of RR Lyrae stars with multiple distinct kinematic components have recently been studied by Kollmeier et al. (2009) and Sesar et al. (2010b). However, these investigations using Sloan Digital Sky Survey (SDSS) data were unable to trace this structure beyond a survey area of $\sim 300 \mathrm{deg}^{2}$ and to a limiting depth of $120 \mathrm{kpc}$. Other surveys of RR Lyrae stars have investigated their distributions to shallower depths though they typically covered larger areas of the celestial sphere. The Southern Edgeworth-Kuiper Belt Object (SEKBO) survey identified RR Lyrae stars within a $1675 \mathrm{deg}^{2}$ region extending out to $\sim 50 \mathrm{kpc}$ (Keller et al. 2008). The Quasar Equatorial Survey Team (QUEST) survey extended farther to $\sim 60 \mathrm{kpc}$ but covered only $\sim 380 \mathrm{deg}^{2}$ (Vivas \& Zinn 2006). The Northern Sky Variability Survey (NSVS) studied RR Lyrae across "twothirds" of the sky but only out to $\sim 9.5 \mathrm{kpc}$ (Kinemuchi 2004; Kinemuchi et al. 2006; Wils et al. 2006). The Optical Gravitational Lensing Experiment (OGLE) survey identified RR Lyrae stars in the Galactic bulge at $~ 8 \mathrm{kpc}$ (Collinge et al. 2006; Pejcha \& Stanek 2009). To more fully compare simulations of hierarchical Galaxy formation to our local environment, we will need to follow filaments of substructure across the entire sky, requiring an increase in the depth and breadth of the known RR Lyrae stars' population.

In the course of its 10 year, six-passband $3 \pi$ survey (Ivezić et al. 2008a), the Large Synoptic Survey Telescope (LSST) is expected to observe of order $10^{5} \mathrm{RR}$ Lyrae stars, uncovering Galactic substructure to beyond the Milky Way tidal radius at $\sim 300 \mathrm{kpc}$ and details of satellite galaxies. The ability of this photometric survey to achieve particular science goals depends on several factors including the observing frequency, survey duration, depth, sky coverage, and photometric precision. The study presented here is designed to explore LSST's capabilities for the recovery of RR Lyrae stars' light curve properties as a function of LSST cadence, a star's distance modulus, and the LSST survey duration. This will in turn provide a figure of merit for LSST's ability to measure Galactic halo substructure, measure the halos and populations of nearby galaxies, and discover new dwarf galaxies throughout the Local Group.

Section 2 discusses the utility of RR Lyrae stars for studies of Galactic structure. We provide a summary of LSST and its observation program in Section 3 along with our methods for realizing simulated LSST surveys of various duration and our process for calculating photometric errors. Sections 4-6 present the techniques that we have utilized to analyze the simulated light curves, including period and flux recovery, stellar typing, and light curve shape determination. The main results of our analyses are discussed in Sections 7 and 8.

\section{RR LYRAE VARIABLE STARS}

\subsection{As Standard Candles}

RR Lyrae stars are evolved horizontal branch stars, and their main-sequence progenitors are thought to be both numerous (with a main-sequence mass of $\sim 0.8 M_{\odot}$ ) and old (with a mainsequence lifetime of $\sim 10$ Gyr; Salaris \& Cassisi 2005). RR Lyrae stars are sufficiently bright $-M_{V} \approx 0.6$ (Chaboyer 1999) for a metallicity of $[\mathrm{Fe} / \mathrm{H}]=-1.5$ (Ivezić et al. 2008a)—to be seen to large distance ( $600 \mathrm{kpc}$ for a survey with limiting magnitude $\sim 24.5$ ). Additional corrections to their absolute magnitude due to both period and metallicity make these standard candles with a small $(\approx 4 \%)$ scatter (Cáceres \& Catelan 2008). They lie within the so-called instability strip for stellar variability, and the more numerous RR Lyrae stars of type $a b$ (RRab) are readily recognizable in time-series data due to their distinct light curve shapes. These characteristics combine to make RR Lyrae stars useful tracers of the old, population II, structure of our Galactic halo (Ivezić et al. 2000, 2005; Sesar et al. 2007, 2010a; Keller et al. 2008; Watkins et al. 2009).

RR Lyrae stars pulsating in the fundamental radial mode are called RR Lyrae stars type $a b$ while stars pulsating in the radial first overtone are designated RR Lyrae stars type $c$ (RRc; Bailey 1902). RRab tend to have asymmetric light curves with amplitudes between 0.2 and $1.5 \mathrm{mag}$ and periods ranging from 0.4 to 1.1 days. RRc light curves are more sinusoidal in shape, have lower amplitudes between 0.1 and $0.8 \mathrm{mag}$, and shorter periods ( 0.2 to 0.5 days). To use these stars as tracers for an underlying stellar population, one needs to be able to recognize them from other types of variability, to determine their pulsational period, to measure their brightnesses, and (optimally) to recover the shape of the light curve to estimate the population's metallicity. Recovering the distances to RR Lyrae stars with the highest accuracy possible requires recovery of their brightnesses to within the systematic uncertainty in their period-brightness calibration $\left(\sigma_{V} \approx 0.03\right.$ mag; Cáceres \& Catelan 2008).

\subsection{Metallicity and Light Curve Shape}

Jurcsik \& Kovács (1996) derived a linear metallicity relationship in the pulsational period $P$ and phase difference between the first and third harmonic $\phi_{31}$ (where $\phi_{n m}=m \phi_{n}-n \phi_{m}$ ) in a Fourier decomposition of RRab $V$-band light curves:

$$
[\mathrm{Fe} / \mathrm{H}]=1.345 \phi_{31}^{(s)}-5.394 P-5.038,
$$

where $s$ indicates that the phases were the result of a sine decomposition and $\phi$ has units of radians. The small root-meansquare (rms) scatter around this relationship, 0.14 dex, suggests that light curve shape can convey information on the stellar metallicity. However, the shape of this relationship (as well as the variance about it) will be different for data in different passbands, thus relationships such as Equation (1) must be estimated independently for observations in LSST passbands.

More appropriate for our study of LSST photometry is the refinement of RRab photometric metallicities developed in the SDSS photometric system by Sesar et al. (2010a), which uses the period $P, g$-band light curve amplitude $A_{g}$, and phase of the third Fourier harmonic $\phi_{31}$ of the decomposed $g$-band light curve to yield the following relation:

$$
[\mathrm{Fe} / \mathrm{H}]=0.313 \phi_{31}^{(s)}-3.65 P-0.493 A_{g}-0.66
$$




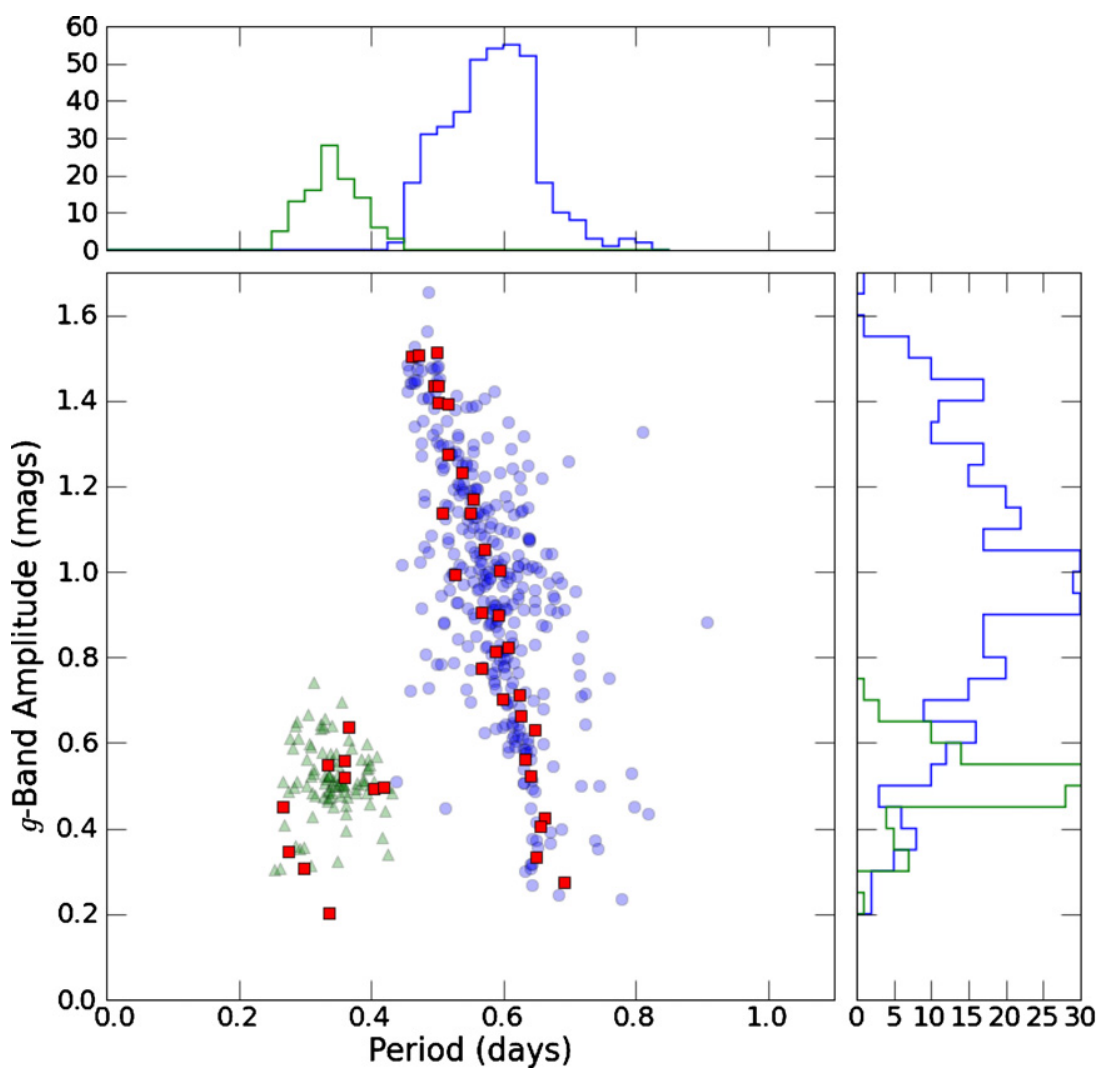

Figure 1. Period-amplitude relationship in the $g$ band for 483 RR Lyrae stars from Sesar et al. (2010a). RRab stars are indicated by the blue circles, while RRc are indicated by the green triangles. The $30 \mathrm{RRab}$ and $10 \mathrm{RRc}$ stars selected for study in this analysis are overplotted as red squares on top of the full distribution and were chosen to span the range of amplitudes and periods. Histograms of the period and amplitude distributions for RRab and RRc stars are shown along the top and right axes, respectively. These distributions are preserved in our simulation by scaling the recovery results as described in Section 4.

(A color version of this figure is available in the online journal.)

When tested against randomly generated $g$-band data, this relation was found to be more robust than the $V$-band relation, but suffers from a large rms scatter of 0.26 dex due to uncertainties in the spectroscopic metallicity measurements. Since there typically will be many more observations taken in the LSST $r$ band compared to the $g$ band, we have determined a similar metallicity measure using the same data as Sesar et al. (2010a), but using the $r$-band light curve shapes. This $r$-band relation for $[\mathrm{Fe} / \mathrm{H}]$ is

$$
[\mathrm{Fe} / \mathrm{H}]=0.175 \phi_{31}^{(s)}-2.29 P-0.301 A_{r}-0.75,
$$

where $A_{r}$ is the $r$-band light curve amplitude and $\phi_{31}$ is derived from a sine decomposition of the $r$-band light curve. This relation possesses an rms deviation of $0.27 \mathrm{dex}$, similar to that of Equation (2). However, the shallower slope on $\phi_{31}$, compared to Equation (2), indicates that shapes in the $r$ band are less sensitive to metallicity. We will use both of these relationships to quantify LSST's ability to recover the metallicities of RRab stars using the shapes of the recovered light curves.

For RRc stars, Morgan et al. (2007) obtained an $[\mathrm{Fe} / \mathrm{H}]$ relationship quadratic in $\phi_{31}$ utilizing a cosine decomposition of $V$-band light curves:

$$
\begin{aligned}
{[\mathrm{Fe} / \mathrm{H}]=} & 0.131 \phi_{31}^{2}+0.982 \phi_{31}^{(c)}-4.198 \phi_{31}^{(c)} P \\
& +52.466 P^{2}-30.075 P+2.424
\end{aligned}
$$

They reported an rms scatter of 0.14 dex for this relationship. It was tested against SDSS $g$-band data by Watkins et al. (2009) who observed an increased scatter of 0.38 dex. Further analysis of RRc ugriz light curve shapes by Sesar et al. (2010a) using 105 RRc stars found that their entire sample was well fit using only two light curve templates per passband. This result suggests that either the light curve shapes of RRc stars do not vary strongly with metallicity or Sesar et al. (2010a) selected a population of RRc stars that fell neatly into two metallicity categories. In either case, photometric metallicity estimates of RRc stars are not well constrained, and accordingly we restrict our analysis to RRab stars.

\subsection{Light Curve Selection}

Sesar et al. (2010a) have provided a catalog of five-band ugriz light curves for 483 RR Lyrae stars from the SDSS's "Stripe 82" region (see, e.g., Adelman-McCarthy et al. 2008). The objects have an average of 30 observations in each of the SDSS ugriz passbands and the RRab and RRc subtypes are distributed roughly in a 3:1 ratio in the SDSS Stripe 82 data set. Figure 1 shows the full distribution of RRab and RRc periods and $g$-band amplitudes from Sesar et al.'s (2010a) data set along with histograms of the period-amplitude distribution of the sample. The period distribution of the RRab stars is peaked at $\approx 0.6$ days and their amplitude distribution is peaked at $\approx 1.0 \mathrm{mag}$. This distribution, made up of both field stars and those in overdensities, represents an intermediate Oosterhoff type with some bias toward Oosterhoff type I. For computational efficiency, we chose to use 40 representative light curves - 30 of type RRab and 10 of type RRc-from among the complete set of 483 that evenly samples the period-amplitude 



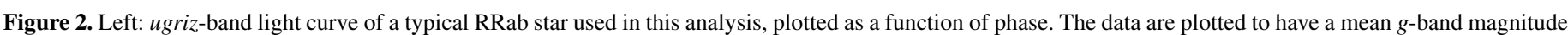

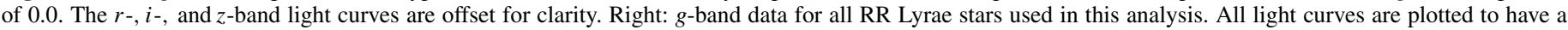
mean $g$-band magnitude of 0.0. RRab-type stars are plotted on the top and RRc-type stars on the bottom with a 1 mag offset for clarity.

(A color version of this figure is available in the online journal.)

distribution of these stars. The 3:1 ratio of $R R a b$ to $R R c$ represents an intermediate Oosterhoff type consistent with the period-amplitude distribution of our stars and also reflects the ratio of RRab to RRc stars in the full sample of 483 stars, which contains 379 RRab stars and 104 RRc stars. The 40 stars we use in our analyses are shown with bold symbols in Figure 1.

In Sesar et al. (2010a) the light curve of each star, and in each passband, was fit with a smooth model that may be used to interpolate the light curve at all phases. These idealized light curves are the inputs used in the analysis described below. Figure 2 provides the ugriz light curves for a particular RRab star used in our analysis (left panel); the color relationships and amplitudes of the five-band light curves are preserved in the simulation. The full set of $g$-band light curves (30 RRab and $10 \mathrm{RRc}$ ) are shown in the right panel to demonstrate the diversity of shapes that exist within this population.

\section{LSST SURVEY SIMULATION}

The LSST is anticipated to undertake a 10 year, $3 \pi$ sr survey that promises to observe millions of new periodic variable stars (Ivezić et al. 2008a). The filter set includes six ugrizy passbands, the first 5 of which are very similar to the SDSS ugriz bands (Fukugita et al. 1996). LSST will extend to fainter magnitudes than many previous time-domain surveys, with single visit depths of $r \sim 24.5$ in $15 \mathrm{~s}$ exposures covering $10 \mathrm{deg}^{2}$. The faint per-exposure limiting magnitude will detect RR Lyrae stars far beyond the observed steepening of the Galactic halo profile at $28 \mathrm{kpc}$ (Sesar et al. 2010a; Deason et al. 2011), and beyond the Galaxy's tidal radius at $300 \mathrm{kpc}$ (Ivezić et al. 2008a).

The core LSST observing strategy is to take two back-toback $15 \mathrm{~s}$ exposures in a given filter (defining a "visit"), and to return to the same pointing within 15-60 minutes (defining a field "revisit;" Ivezić et al. 2008a). The choice of filter is driven by airmass, sky-brightness and seeing considerations, as well as by final co-added depth-per-filter requirements. This Universal Cadence (UC), which will consume the majority ( $90 \%)$ of LSST's observing time, is designed to image $\sim 20,000 \mathrm{deg}^{2}$ of the sky with a total of $\sim 1000$ observations per field, distributed among all filters. The baseline specifications for the number of field revisits in the ugrizy passbands are 70, 100, 230, 230, 200, and 200, respectively, yielding the most temporal sensitivity in the $r$ - and $i$ bands. However, there are other planned observing modes which we will examine in the context of studying RR Lyrae stars' variability.

The process of simulating realizations of LSST-observed RR Lyrae stars' light curves is done using two levels of simulation. First, we use a cadence simulation that spans the planned 10 year lifetime of LSST. This describes the particular realization of an observing strategy that is optimized to satisfy the various LSST science goals. We then implement a light curve simulation that takes the smooth idealized light curves described in Section 2.3 and synthesizes photometric observations of them at each epoch based upon the simulated observing conditions. We describe each step in detail below.

\subsection{Cadence Simulation}

The LSST Project has developed an operation simulator to investigate how best to observe the sky to achieve its multiple science goals (Cook et al. 2004). The simulator has a sophisticated model of the telescope and dome to properly constrain potential observing sequences. The simulator balances cadence goals from multiple science programs and attempts to minimize time spent slewing as it carries out these goals. Ten years of LSST operations have been repeatedly simulated using realistic seeing distributions, historical weather data, scheduled engineering downtime, and current telescope and camera parameters. This study uses the particular cadence simulation version opsim1_29, which is the reference simulation for version 1.0 of the LSST Science Book.

In this paper, we investigate observations from five distinct LSST observing modes: the aforementioned UC deep-wide-fast strategy; a North Ecliptic extension optimized for solar system (SS) observations and is thus undertaken at higher airmass than the UC fields; Milky Way (MW) observations which allocate 30 observations per filter to $10^{3} \mathrm{deg}^{2}$ around the Galactic center; and Deep Drilling (DD) observations which allocate 10 minutes of continuous exposure per night, distributed among filters on a five day cycle. We also examine the overlap (OL) regions between adjacent UC fields. Tables 1-3 provide statistics on the average number of field visits per year in the gri bands for 




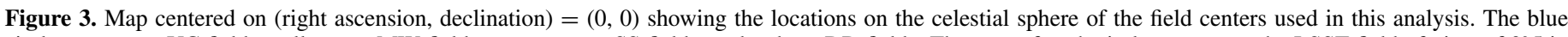

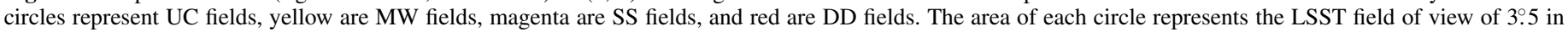
diameter. The dots represent OL positions.

(A color version of this figure is available in the online journal.)

Table 1

Average Number of $g$-band Field Visits per Season in opsim1_29

\begin{tabular}{lrrrrrrrrrr}
\hline \hline Cadence & 1 & 2 & 3 & 4 & 5 & 6 & 7 & 8 & 9 & 10 \\
\hline DD & 317 & 265 & 272 & 280 & 289 & 297 & 241 & 274 & 255 & 278 \\
MW & 8 & 17 & 4 & 0 & 0 & 0 & 0 & 0 & 0 & 0 \\
SS & 0 & 2 & 1 & 1 & 1 & 1 & 1 & 1 & 0 & 0 \\
UC & 12 & 14 & 9 & 11 & 11 & 13 & 11 & 13 & 11 & 12 \\
\hline
\end{tabular}

Table 2

Average Number of $r$-band Field Visits per Season in opsim1_29

\begin{tabular}{lrrrrrrrrrr}
\hline \hline Cadence & 1 & 2 & 3 & 4 & 5 & 6 & 7 & 8 & 9 & 10 \\
\hline DD & 593 & 517 & 532 & 552 & 570 & 598 & 487 & 572 & 520 & 601 \\
MW & 9 & 16 & 3 & 0 & 0 & 0 & 0 & 0 & 0 & 0 \\
SS & 2 & 3 & 3 & 4 & 4 & 3 & 3 & 3 & 2 & 2 \\
UC & 13 & 19 & 28 & 27 & 31 & 28 & 29 & 29 & 29 & 30 \\
\hline
\end{tabular}

LSST's four main cadences: DD, MW, SS, and UC. The OL fields do not have distinct field pointings in the database, but are typically sampled at twice the UC rate.

In total we have selected 1007 field centers in which to realize each of our 40 RR Lyrae stars' input light curves, such that a statistically relevant sampling of each LSST observing mode is realized. These include 400 UC fields, 400 OL fields, $100 \mathrm{MW}$ fields, $100 \mathrm{SS}$ fields, and all 7 of the opsim1_29 DD fields. Selecting multiple field centers per observing mode averages over nuisance parameters such as weather and the zero point of phase in an RR Lyrae star's pulsation. Figure 3 shows the locations of the various field centers used in our analysis mapped onto the celestial sphere.

\subsection{Light Curve Simulations}

The light curve simulator aggregates information from the operation simulator and from the 40 idealized light curves described in Section 2 to produce realistic time-series light curves of each object. Because SDSS only observed in ugriz, we use the SDSS $z$-band light curves to simulate the LSST $y$-band data (i.e., $z-y=0$ at all epochs). This choice has minimal effect on our simulation despite the fact that one may expect the amplitude of variation to be lower in the $y$-band than in the $z$-band. These bands are expected to be in the Rayleigh-Jeans tail regime of the spectral energy distributions of these stars and
Table 3

Average Number of $i$-band Field Visits per Season in opsim1_29

\begin{tabular}{lrrrrrrrrrr}
\hline \hline Cadence & 1 & 2 & 3 & 4 & 5 & 6 & 7 & 8 & 9 & 10 \\
\hline DD & 622 & 528 & 533 & 553 & 573 & 603 & 486 & 553 & 513 & 565 \\
MW & 9 & 17 & 2 & 0 & 0 & 0 & 0 & 0 & 0 & 0 \\
SS & 2 & 2 & 2 & 4 & 3 & 4 & 5 & 2 & 2 & 2 \\
UC & 17 & 20 & 24 & 28 & 27 & 32 & 26 & 25 & 28 & 27 \\
\hline
\end{tabular}

we do not utilize the $y$-band data's amplitude in any part of our analysis.

The simulator interacts with an SQL database containing the observation parameters for all pointings in realization opsim1_29 of the operation simulator (OpSim). Each input light curve is first initiated at an initial random phase and interpolated with a spline. This spline is sampled based on the observation times provided by the OpSim database for each field center. If the input light curve is periodic, as is the case with RR Lyrae stars, the light curve is replicated indefinitely to cover the span of the survey.

A simulated observation consists of a measured magnitude for the variable source and the associated error in magnitude. Magnitudes provided to the user are Asinh magnitudes (see Lupton et al. 1999). To provide realistic observed light curves, the simulator adds random noise to the observed magnitudes based on the calculated error for that observation. Errors are calculated in two ways depending on how close the object is to the detection limit. For detections that are $10 \sigma$ above the background or better, the errors as a function of magnitude, $m$, are assumed to be symmetric and are calculated based on the relation

$$
\epsilon(m)=\sqrt{(0.04-\gamma) x+\gamma x^{2}}
$$

where $x=10^{0.4\left(m-m_{5 \sigma}\right)}$. The parameter $\gamma$ is band specific and ranges from 0.037 to 0.040 (Ivezić et al. 2008a). The $5 \sigma$ limiting magnitude per observation is denoted $m_{5 \sigma}$ and is provided by the operation simulator. Figure 4 shows the random photometric errors $\epsilon(m)$ computed from Equation (5) as a function of apparent stellar magnitude. The value of $\epsilon$ is added in quadrature with the systematic error for LSST, assumed to be $0.01 \mathrm{mag}$, to obtain the final error in the simulated magnitude measurement. In cases where the simulated detection is $10 \sigma$ or less, asymmetric errors in magnitude are calculated from the $1 \sigma$ flux derived from the $5 \sigma$ limiting magnitude per 


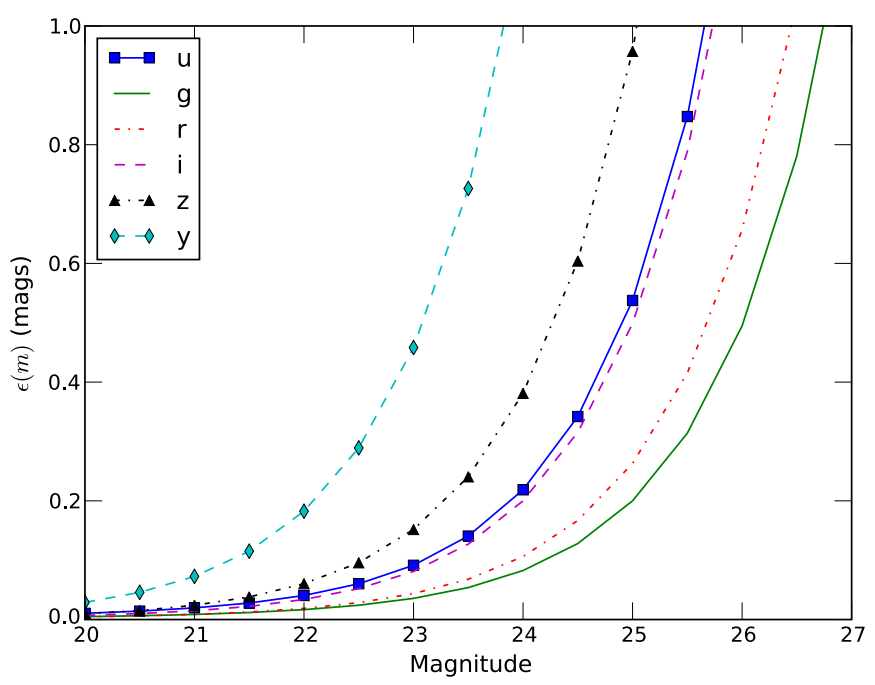

Figure 4. Band-specific LSST random photometric errors as a function of magnitude $\epsilon(m)$ as calculated from Equation (5).

(A color version of this figure is available in the online journal.)

observation. When simulating the scatter in measurement due to the photometric error, the deviation is drawn from a normal distribution scaled to the $1 \sigma$ flux. Figure 5 shows one season (200 days) of observations for a UC field center (left) and DD field center (right). All six passbands of data are presented with error bars for each data point.

Some assumptions are inherent in this technique for simulating observed time series of idealized light curves. Since the objects are placed on the sky with no contributing context, real world complications like overlapping point spread functions, ghosting, and scattered light effects cannot be taken into account. These issues could potentially be significant, especially in crowded fields, but will require measurement on simulated images to address properly. There is also an assumption that the light curves do not evolve over the course of the survey. This could, in principle, be simulated by hand, but is not a feature of the current simulator. Additionally, there is no model currently in place in the simulator to allow for drift in measurement accuracy that would introduce correlation in the errors.

To explore LSST's ability to recover light curve shape information as a function of distance, we realize each idealized light curve used in the simulations over a range of mean $g$ band magnitudes, from 20.0 to 27.0 in steps of 0.5 mag. While the bright limit is $g \sim 16$, the simulations start at $g=20.0$ as the LSST photometric errors for $g<20$ will be roughly constant (Ivezić et al. 2008a). Finally, we explore the evolution of LSST's state of knowledge of each star by exploring subsets of the 10 year simulated light curves in 1 year increments, which may be related to the number of epochs in each cadence and filter via the information in Tables 1-3. In total, this yields 40 RR Lyrae stars' light curves $\times 6$ filters $\times 1007$ field centers $\times 15 \mathrm{~g}$-band magnitude bins $\times 10$ sub-surveys of light curve information, for just over 36 million light curves that have gone into this study.

\section{PERIOD RECOVERY}

\subsection{Defining Period Recovery}

Among the first steps in classifying variability is searching for periodicity in the observed light curve. To this end we have run period-finding software on each of our $\sim 36$ million simulated light curve realizations. We used the variable span Supersmoother algorithm of Reimann (1994) for period estimation. Supersmoother is able to uncover a variety of light curve shapes since it makes no explicit assumptions about the underlying shape of the curve, only that when folded it be smooth and continuous. In our implementation, Supersmoother searched for periods as small as 0.1 days and as long as the temporal length of each light curve, mimicking a "blind" search through the data for variability (systems whose periods are equal to the temporal length of each light curve are likely aperiodic systems varying coherently throughout the data set). It is possible that improvements in the efficiency of the period recovery might be attained by limiting the period searches to the range spanned by known RR Lyrae stars. Nonetheless,
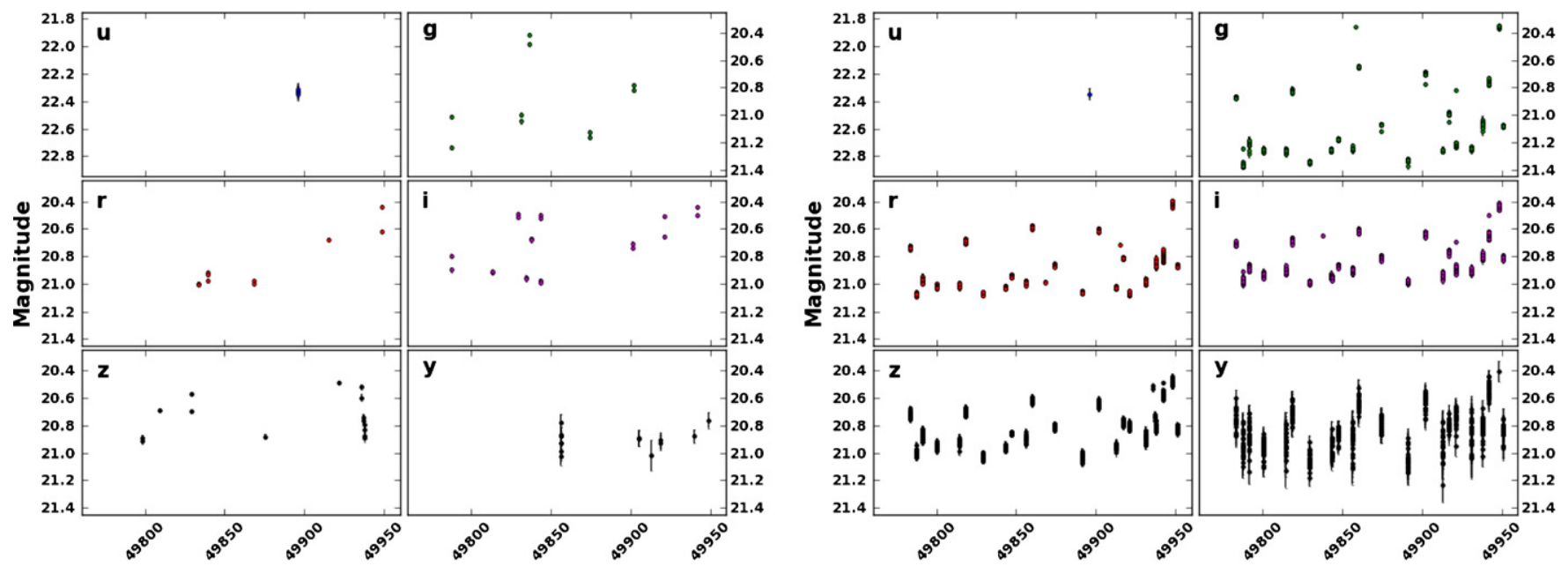

Figure 5. Two hundred days of simulated LSST observations of the RR Lyrae star's template shown in Figure 2, with a magnitude offset such that the mean $g$-band magnitude is 21. The left panel shows a field sampled at the Universal Cadence (UC), while the right panel shows a neighboring field sampled at the Deep Drilling cadence (DD). For these particular fields, the DD cadence yields a factor of $\sim 20$ more observations than in the UC fields. To illustrate the differences in observing modes, and to demonstrate how the quality of the light curves decreases with increasing mean magnitude, we plot in Figure 7 the full 10 year $g$-band data set for the same star shown in Figure 5, folded at its correct period. The panel on the left is for a field sampled at the UC cadence, and on the right at a DD cadence. It is clear that the DD light curves are more densely sampled, but also sample a larger range of observing conditions since there are many points with large scatter and error bars.

(A color version of this figure is available in the online journal.) 
the periods recovered would be identical to those obtained in the blind search.

The precision of the recovered period typically improves with survey length. However, it also depends on details of LSST's observation cadence, including the number of light curve samples, and the phase distribution of the samples. To ascertain whether or not Supersmoother recovered the known input period, we define a matching criteria to delineate period recovery using the quality of the period-folded data as a metric. We require that the product of the fractional misfit in the recovered period and the number of pulsation cycles be less than some fraction of a cycle:

$$
N \times \sigma_{P} \leqslant \delta \phi_{\max }
$$

where $\sigma_{P} \equiv\left|P_{\mathrm{SS}}-P_{\text {in }}\right| / P_{\text {in }}, P_{\text {in }}$ is the known input period, and $P_{\mathrm{SS}}$ is the fitted Supersmoother period. The number of cycles $N$ is given by the ratio of the survey length $\Delta t$ and the period of variability $P_{\text {in }}$, and $\delta \phi_{\max }$ is the maximum allowed phase offset after period-folding $N$ cycles. Our criterion for period recovery is thus given by,

$$
\frac{\left|P_{\mathrm{SS}}-P_{\text {in }}\right|}{P_{\text {in }}} \leqslant \frac{\delta \phi_{\max } P_{\text {in }}}{\Delta t} .
$$

This criterion on the fractional misfit in period is generally appropriate for stars that are variable on multiple timescales, since it provides a tighter matching criterion for shorter period stars. For a given number of observations taken over a finite window in time, these shorter period stars will have gone through more oscillations, and should have a more tightly constrained period.

To estimate a reasonable value for $\delta \phi_{\max }$, we folded several 10 year light curves with varying misfits $\delta P$ on the period (where $\left.\delta P \equiv \sigma_{P} / P_{\text {in }}=\delta \phi_{\max } / \Delta t\right)$. Values of $\delta \phi_{\max } / \Delta t<10^{-5} \mathrm{day}^{-1}$ (or $\delta \phi_{\max }=0.037,1 / 27$ th of a cycle) were found to yield light curves with well-resolved minima in the RRab stars. Thus, in terms of the input period $P_{\text {in }}$ and the recovered period $P_{\mathrm{SS}}$, our criterion for successful period recovery may be written as

$$
\frac{\left|P_{\mathrm{SS}}-P_{\text {in }}\right|}{P_{\text {in }}^{2}} \leqslant 10^{-5} \mathrm{day}^{-1} .
$$

Shown in Figure 6 is the evolving requirement on the relative uncertainty $\sigma_{P}$ that ensures a maximum period-folded phase offset of less than $1 / 27$ th of a cycle. For a star with a period of 0.5 days, this criterion defines a successful period measurement to be within $\approx 0.22$ s of the true period for a 10 year survey.

The criterion defined in Equation (8) for a 10 year survey was applied to periods recovered from the $g, r$, and $i$ passbands. Data in these passbands typically have higher signal-to-noise and dense phase coverage. A successful period recovery is thus defined by having at least two out of these three periods within the tolerance specified in Equation (8). This criterion, which uses multiple passbands of information, is designed to simulate the likely approach an observer would take to identify periodicity given an ensemble of photometric data. Figure 7 shows the entire $10 \mathrm{yr} g$-band light curves of simulated LSST observations of RR Lyrae folded with the best-fit period determined via the process described above.

\subsection{Impact of Aliasing on Period Recovery}

The effects of the 1 day sampling alias on the recovery of the RR Lyrae periods are examined here. If Equation (8) is evaluated

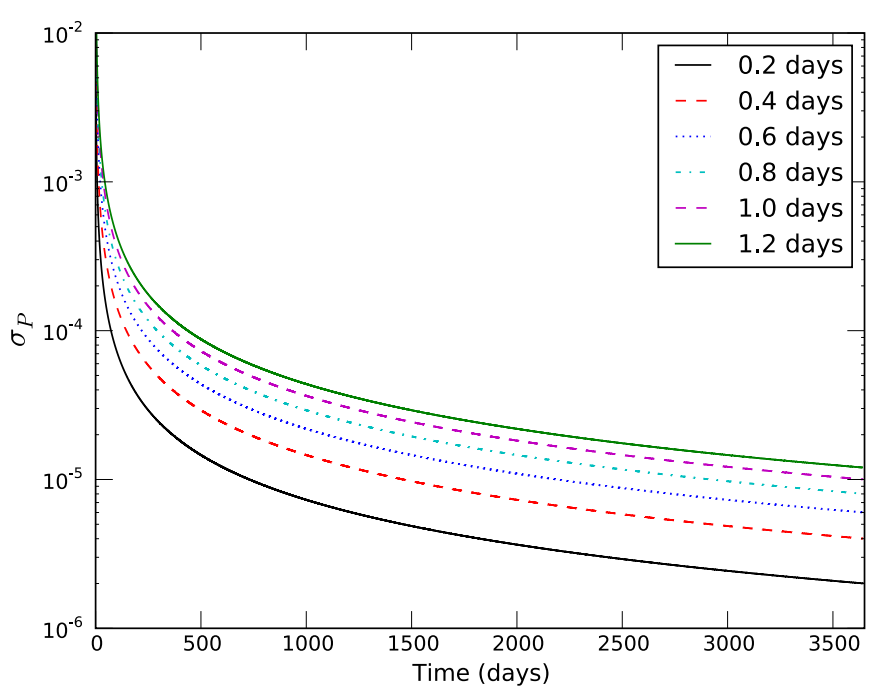

Figure 6. Evolving criterion on the relative uncertainty in the recovered period $\sigma_{P}$ as a function of survey length and stellar period is shown above. The criteria are calculated as the minimum precision for phasing all data to within $1 / 27$ th of a cycle. RR Lyrae stars with the minimum period of 0.2 days require a relative uncertainty better than $2 \times 10^{-6}$ while RR Lyrae stars with periods 1.0 days or longer require a precision better than $1 \times 10^{-5}$.

(A color version of this figure is available in the online journal.)

with $P_{\text {in }}=1.0$ days, which reflects the sensitivity of our period recovery to this alias, we find that no stars match this criteria in two of the three gri passbands, even though we do find stars that in a single passband have periods within $10^{-5}$ of 1.0 days. This indicates that our period recovery criteria are stringent enough, and that the LSST cadence is varied enough within and between passbands, to avoid classical sampling aliases. If we loosen our period recovery criteria to allow matches within $10^{-3}$ of the 1 day alias, approximately $0.5 \%$ of stars in the UC fields have at least one passband's worth of data whose best-fit period matches the sampling alias in the first year of the survey, decreasing to $0.2 \%$ by year 2 , and yielding only a residual $0.1 \%$ aliased population after 10 years between $24.5<g<27.0$. However, even with this relaxed recovery criterion, only a handful of objects throughout the entire sample have this period alias in two of the three gri passbands, indicating that this alias should not play a significant role in period recovery for LSST, especially if data from multiple passbands are used in the period assessment.

\subsection{Light Curve Shape and Period Recovery}

Figure 8 shows the average magnitude where the period recovery efficiency drops below $90 \%$ for 10 year UC and DD observations versus the amplitude of the $g$-band brightness variation for all 40 of our input stars. As expected, this recovery varies substantially with the amplitude of variation such that the greater the amplitude the dimmer the stars that can be well characterized. Since our subsample of 30 RRab stars and 10 RRc stars evenly samples the period-amplitude space represented by the full distribution, these results do not reflect any particular Oosterhoff phase-amplitude-population space.

It is interesting to note the differences in recovering the periods of RRab and RRc stars as separate populations. This will provide some insight into the effect of light curve shape on period recovery. Figure 8 shows that for equivalent amplitudes of variation, the RRab periods are recovered for dimmer stars in all cases. Type RRab stars possess faster rise times and as a result, more sharply peaked light curves as compared to RRc. 

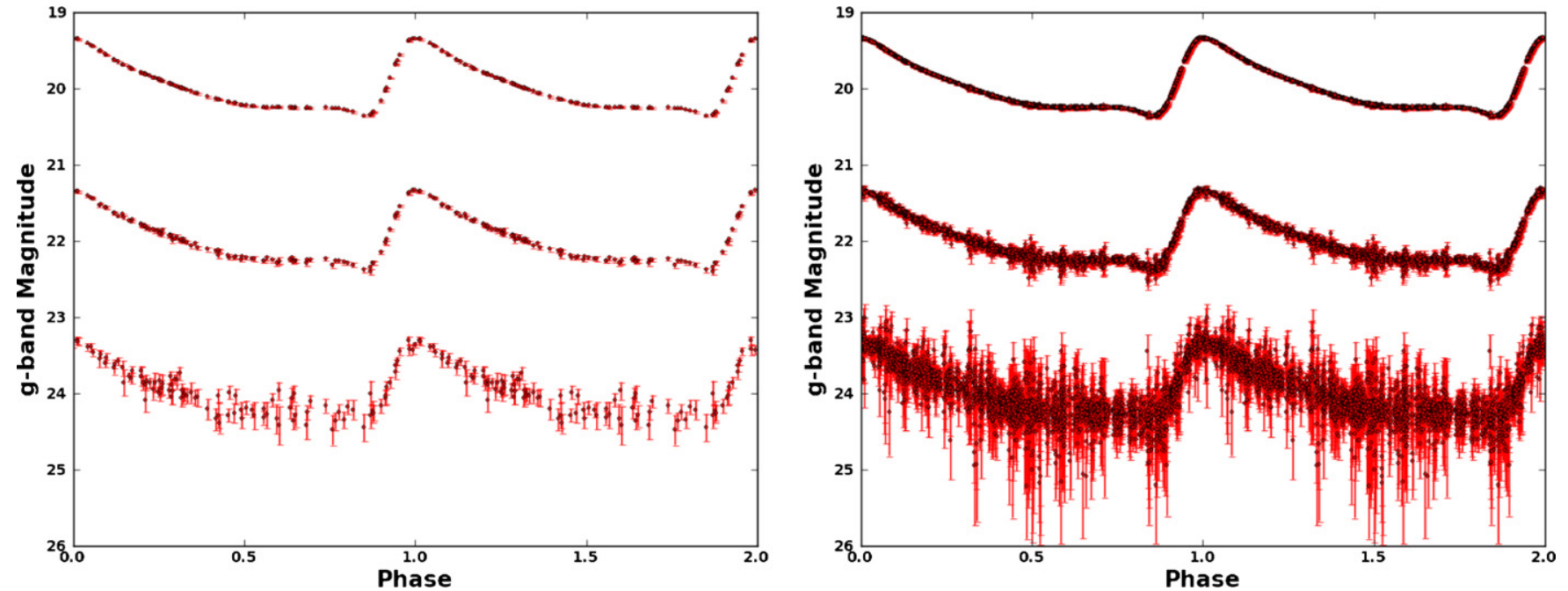

Figure 7. Entire 10 year $g$-band light curve of simulated LSST observations of the RR Lyrae star's template shown in Figure 2, folded at its period of 0.594659 days. The left panel shows a field sampled at the Universal Cadence (UC), while the right panel shows a neighboring field sampled at the Deep Drilling cadence (DD). We show light curves for stars having mean $g$-band magnitudes of 20,22, and 24. The apparent photometric scatter in the DD light curves is seemingly much larger than in UC light curves due to a much larger number of data points which sample a wider range of atmospheric conditions.

(A color version of this figure is available in the online journal.)
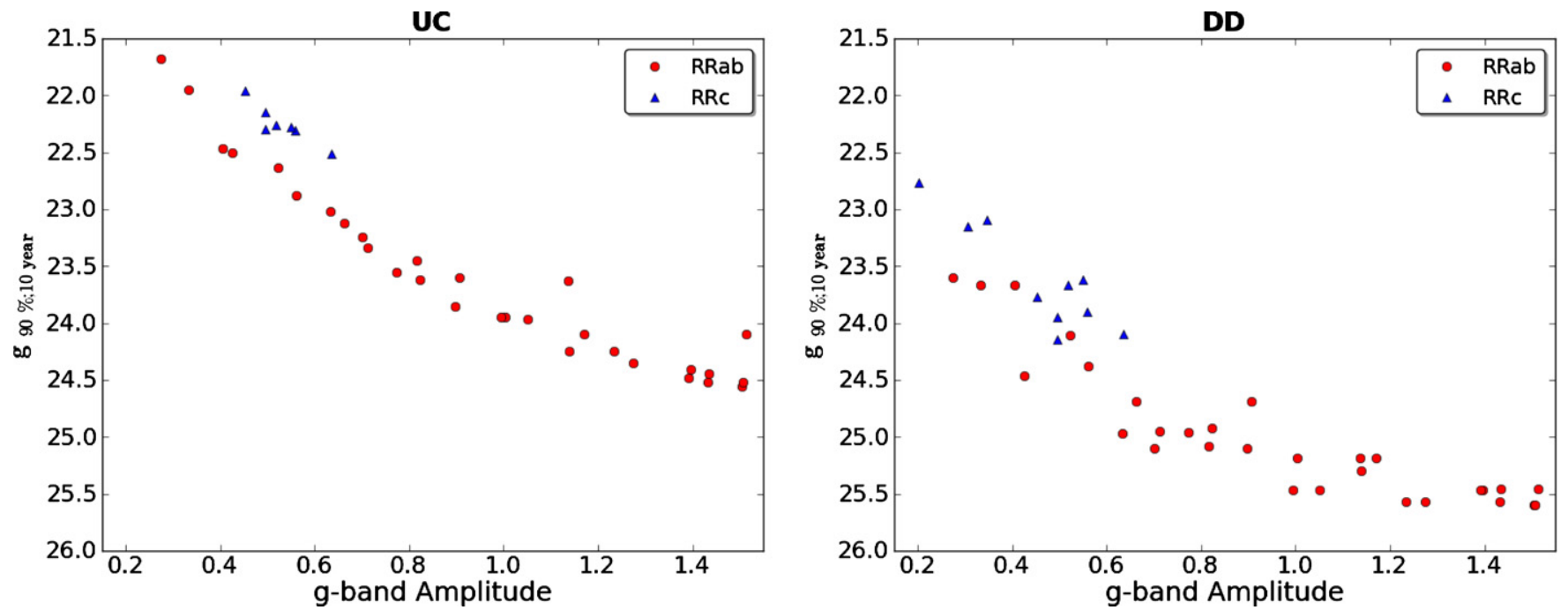

Figure 8. Plot of $g$-band magnitude where the period recovery efficiency falls below $90 \%$ after the full 10 years of LSST as a function of $g$-band amplitude. The red circles are for RRab stars and the blue triangles are for RRc stars. The left panel shows this averaged over all UC fields, and the right panel shows this averaged over all DD fields.

(A color version of this figure is available in the online journal.)

This result is not unexpected as the period of a phenomenon may be determined to the maximum precision for the ideal case of a periodic delta function. However, in that extreme one may miss many cycles but with the probability of detection increasing with the number of epochs. For the durations of the maxima and minima of RR Lyrae stars' light curves this is not a problem. However, the results shown in Figure 8 are biased by the fact that 10 year light curves were used and thus the number of epochs is large. In the opposite extreme, where the number of epochs is small, one expects that the period of RRc stars would have their periods recovered more efficiently due to their longer duration maxima.

\subsection{Impact of Apparent Magnitudes on Period Recovery}

The median value of $\delta P \equiv \sigma_{p} / P_{\text {in }}$ as a function of stellar magnitude for the combined unweighted set of RRab and RRc stars is shown in Figure 9 for the UC and DD fields (rows) and for the $g$ and $r$ passbands (columns). The horizontal line at $\delta P=10^{-5}$ illustrates our criterion for successfully recovering the period of pulsation.

The additional UC observations in the $r$ band (compared to the $g$ band) yield an improvement in the $\delta P$ distribution in years 1 and 2 of the survey, but by year 5 there are enough data in both passbands to yield essentially equivalent recovery. In the DD fields the numbers of observations are similar in $g$ and $r$, yielding similar recovery profiles. Half of all RR Lyrae stars will have their periods recovered to within $\delta P=10^{-5}$ out to $\langle g\rangle=25.5$, or $\approx 950 \mathrm{kpc}$, in the DD fields, while UC observations will extend to $\langle g\rangle=24.0$, or $\approx 480 \mathrm{kpc}$. We refine these estimates in Sections 4.5 .1 and 4.5 .1 by looking at the $\delta P$ distribution for the nature-provided period-amplitude distribution in greater detail. 



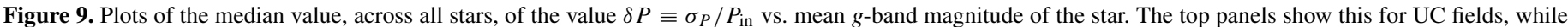

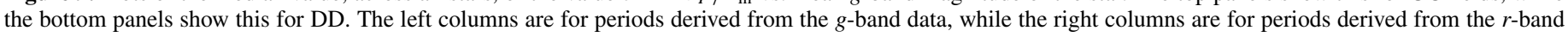
data.

(A color version of this figure is available in the online journal.)

\subsection{Impact of LSST Cadence on Period Recovery}

To ascertain LSST's ability to recover RR Lyrae stars' periods, we mimic the period-amplitude distribution of the full set of 483 stars by weighting the 40 stars in our subsample according to their representation in the amplitude histograms shown in Figure 1. The weighting is done separately for RRab and RRc stars. For each distribution, the probability $P_{b}$ of a star being in a particular amplitude bin of the amplitude histogram (where $b=1 \ldots m, m=$ total number of bins) is given by the number of stars in the bin $n_{b}$ divided by the total number of stars in the distribution $N$. The number of stars in our subsample we denote $N_{s}$, which equals 30 and 10 for RRab and RRc stars, respectively. We denote the number of stars in each amplitude bin as $s_{i}$. The normalized weights $w_{i}$ of each subsample star $i$, where $i=1 \ldots N_{s}$, is then given by

$$
w_{i}=\frac{P_{b}}{s_{i}}=\frac{n_{b}}{s_{i} N}, \quad\left(\sum_{i=1}^{N_{s}} w_{i}=1\right) .
$$

The fraction of stars recovered by a particular cadence mode is obtained from the ratio of the summed weights of successfully recovered stars and the number of fields $N_{f}$ used:

$$
\text { Recovery Efficiency }=\frac{\sum_{k=1}^{N_{f}} \sum_{i=1}^{N_{s}} w_{k i} \delta_{i j}}{N_{f}},
$$

where $\delta_{i j}$ is the Kronecker delta and $j=i$ if a star's property (e.g., period) is successfully recovered and $j=0$ otherwise, and the index $k$ spans the number of fields.

A summary of the $g$-band magnitude below which the weighted period recovery falls to $97.5 \%, 90 \%, 50 \%, 25 \%$, and $10 \%$, for all survey modes using multiple passbands of data, is given in Tables 4 and 5 for RRab and RRc stars, respectively.

\subsubsection{Opsim 1_29 Universal Cadence}

Covering $\sim 20,000 \mathrm{deg}^{2}$ and consuming $90 \%$ of LSST's observing time, the UC and its associated overlap (OL) regions constitute the bulk of LSST data. Shown in Figure 10 are the plots of period recovery for the UC and OL fields. The plots provide the $1,2,5$, and 10 year recovery curves and show the results for RRab stars as solid lines and the results for RRc stars as dashed lines. Since the recovery rate is passband and light 

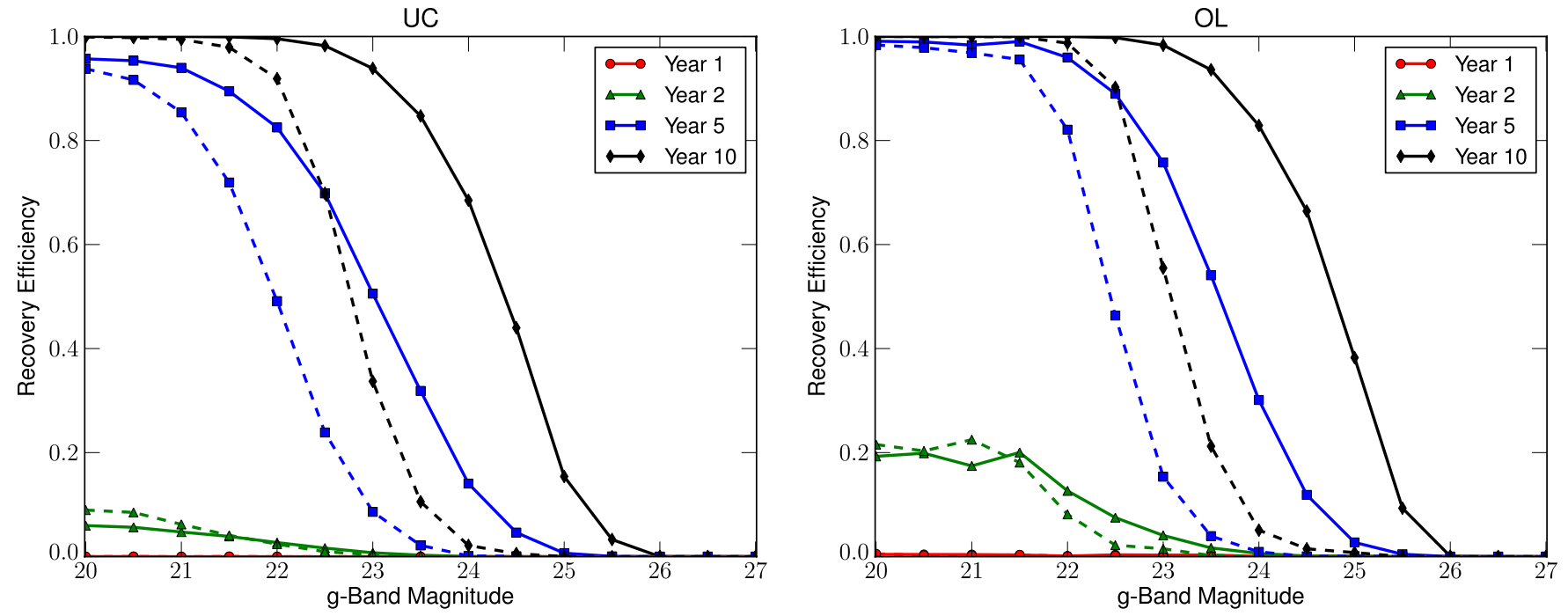

Figure 10. Recovery curves of UC (left) and OL (right) fields after 1, 2, 5, and 10 years of LSST observations. The solid lines indicate the results for RRab stars and the dashed lines indicate the results for RRc stars.

(A color version of this figure is available in the online journal.)

Table 4

RRab Period Recovery $\langle g\rangle$ Mag Limits for a 10 year Survey

\begin{tabular}{lccccc}
\hline \hline Observing & \multicolumn{5}{c}{$a b$ stars period recovery efficiency } \\
Cadence & $97.5 \%$ & $90 \%$ & $50 \%$ & $25 \%$ & $10 \%$ \\
\hline UC & 22.8 & 23.4 & 24.5 & 24.9 & 25.3 \\
OL & 23.1 & 23.7 & 24.8 & 25.2 & 25.5 \\
DD & 24.6 & 25.0 & 25.6 & 25.8 & 25.9 \\
MW & $\ldots$ & $\ldots$ & $\ldots$ & $\ldots$ & 21.4 \\
SS & $\cdots$ & $\ldots$ & 20.2 & 22.2 & 23.0 \\
\hline
\end{tabular}

Table 5

RRc Period Recovery $\langle g\rangle$ Mag Limits for a 10 year Survey

\begin{tabular}{lccccc}
\hline \hline Observing & \multicolumn{5}{c}{$c$ stars period recovery efficiency } \\
Cadence & $97.5 \%$ & $90 \%$ & $50 \%$ & $25 \%$ & $10 \%$ \\
\hline UC & 20.8 & 21.6 & 22.6 & 23.1 & 23.5 \\
OL & 22.1 & 22.5 & 23.1 & 23.4 & 23.8 \\
DD & 22.9 & 23.2 & 24.2 & 24.7 & 25.2 \\
MW & $\ldots$ & $\ldots$ & $\ldots$ & $\ldots$ & 21.5 \\
SS & $\ldots$ & $\ldots$ & 20.5 & 21.7 & 22.3 \\
\hline
\end{tabular}

curve shape dependent, the recovery rates are expected to be much higher in $r$ and $i$, much less in $u$ and $y$, and greater for RRab than for RRc. As expected, RRc stars are recovered more efficiently than RRab stars at brighter magnitudes and during the first two years of the survey due to the differences in the light curve shapes between the two types. After 5 years, the RRab and $\mathrm{RRc}$ recovery is nearly identical for all stars out to $\sim 75.8 \mathrm{kpc}$ $(\langle g\rangle=20.0)$. Beyond this distance, the periods of RRab stars are successfully recovered much more efficiently than for RRc stars due to their larger magnitudes of variation, with the discrepancy being greatest in the range $22.0<\langle g\rangle<23.5$.

Comparison with OL fields shows that after two years, periods are recovered at twice the efficiency as in the UC fields. Overlap regions will have $\geqslant 20 \%$ recovery out to $\sim 120 \mathrm{kpc}(\langle g\rangle=$ 21.0 ) as compared to $\sim 10 \%$ UC recovery. The $10 \%$ recovery efficiency for OL fields extends to $\sim 190 \mathrm{kpc}(\langle g\rangle=22.0)$. The recovery in the UC fields improves substantially after 5 years of operations, with nearly $95 \%(100 \%)$ recovery in the UC (OL) fields in that same magnitude range. Doubling the length of



Figure 11. Recovery curves of DD fields after 1, 2, 5, and 10 years of LSST observations. The solid lines indicate the results for $a b$ stars and the dashed line indicates the results for $c$ stars.

(A color version of this figure is available in the online journal.)

the survey pushes a given completeness level approximately 1.5 mag deeper in both the UC and OL fields. For stars fainter than $\langle g\rangle=22.0$ the recovery efficiency for the 10 year light curves is $\sim 30 \%$ to $60 \%$ better than for the 5 year light curves, decreasing to zero at $\langle g\rangle \approx 26.0$ for both the UC and OL fields.

\subsubsection{Opsim 1_29 Deep Drilling Cadence}

The DD fields, which constitute $70 \mathrm{deg}^{2}$, are visited most frequently among LSST's four cadences, with 40 total observations per night. Figure 11 shows the frequency with which the period recovery criterion was met as a function of LSST survey length and the stars' mean $g$-band magnitudes. By the end of the first year, $\approx 20 \%$ of RRab stars within $\sim 190 \mathrm{kpc}(\langle g\rangle=22.0)$ will have their periods recovered to within $10^{-5}$ by LSST photometry. For RRc stars, a similar amount of completeness is achieved to $\sim 120 \mathrm{kpc}(\langle g\rangle=21.0)$. By year two, the $50 \%$ completeness level extends to just beyond $\sim 300 \mathrm{kpc}(\langle g\rangle=23.0)$ for RRab 

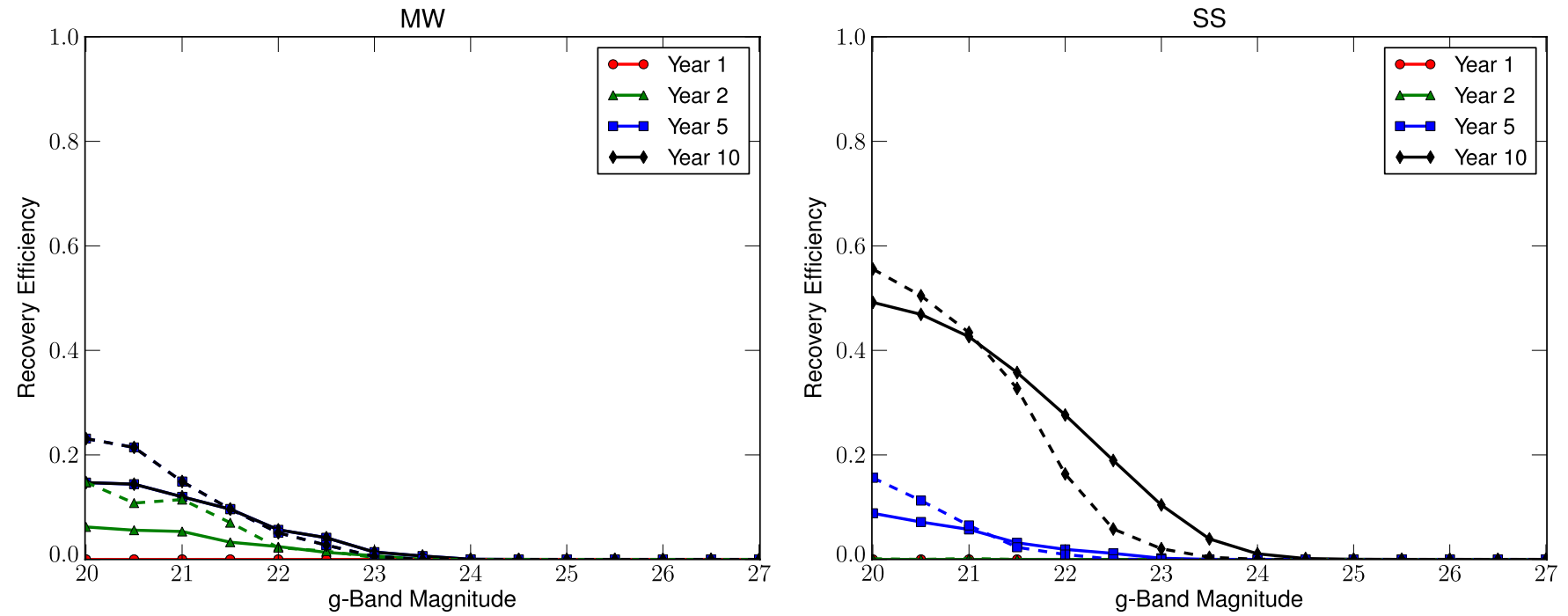

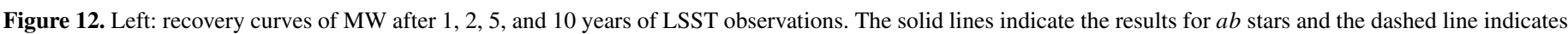

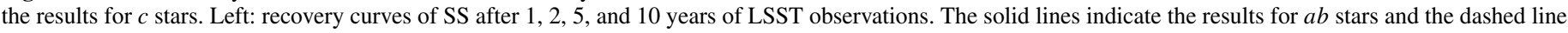
indicates the results for $c$ stars.

(A color version of this figure is available in the online journal.)

stars and just beyond $\sim 190 \mathrm{kpc}(\langle g\rangle=22.0)$ for RRc stars. After 10 years the $50 \%$ recovery extends to $\sim 950 \mathrm{kpc}(\langle g\rangle=25.5)$ for RRab stars and $\sim 550 \mathrm{kpc}(\langle g\rangle=24.3)$ for RRc stars. These results show that LSST will yield the opportunity to detect substructure through RR Lyrae stars' overdensities throughout the Local Group. The recovery or RRab stars' periods falls to nearly zero by $\sim 1.2 \mathrm{Mpc}(\langle g\rangle=26.0)$.

It is interesting to compare period recovery in UC fields to the period recovery in the DD fields. Comparison of the left panel of Figure 10 with Figure 11 illustrates that 2 years of DD data are sufficient to recover periods with the nearly same efficiency as 5 years of UC data (the same is true for 5 years of DD data and 10 years of UC data). However, 5 years of UC data typically contain fewer than $5 \%$ the number of field visits as 2 years of DD data. Apparently, the observational cadence of the UC fields (i.e., back to back $15 \mathrm{~s}$ observations separated by 30 minutes) is much more efficient at recovering RR Lyrae stars' periods than the cadence of the DD fields. The DD fields take 10 minutes of continuous $15 \mathrm{~s}$ observations that are all clustered near the same phase, whereas the UC observations sample a greater phase range. If the DD cadence is modified to sample a greater phase range on a given night, then the efficiency of period recovery for DD fields should increase more rapidly as a function of survey length. One possible approach would be to accumulate a night's allocated observations in two sets of field visits, with a revisit timescale of 30 minutes. This will increase the efficiency of period recovery in DD fields and may also minimize overheads on the overall observing frequency, including minimizing filter changes. After two years, the majority of detectable halo RR Lyrae stars in the field will be measured and the field center could be shifted to a new target. While the recovery of RR Lyrae stars at Mpc distances will require a minimum of 5 years of DD observations, the strategy outlined above would make this process more efficient.

\subsubsection{Opsim 1_29 Galactic Plane Cadence}

Shown in Figure 12 is the period recovery efficiency for MW observations. The RRab/RRc period recovery at $\langle g\rangle=20.0$ is $\approx 7 \% / 16 \%$ for the 2 year survey and $\approx 16 \% / 24 \%$ for the
10 year survey. The recovery rates for both RRab and RRc are equal at $2 \%$ and $6 \%$ by $\langle g\rangle=22.0$ for the 2 year and 10 year surveys, respectively. These low recovery rates result for several reasons. Because of the confusion limit in the Galactic plane, MW observations are restricted to only 30 per filter over the 10 year LSST survey. Also, the cadence of LSST is not optimized to recover the periods of objects with small numbers of observations, since many of the observations are grouped near to each other in time (e.g., for cosmic-ray rejection, and to recover the instantaneous motion vectors of asteroids). In the UC fields, for example, fully half of the field revisits in a given filter occur within 0.02 days, and three quarters of the field revisits happen within 0.06 days. For objects with periods considerably longer than this interval, the visit times are too correlated to be considered independent samplings in phase. Moreover, an investigation of the MW cadence showed that the opsim1_29 strategy was not optimized, with all the allocated MW observations taking place in the first three years of the survey. The priority of the MW fields was being boosted the closer they came to completion, giving them higher priority faster than the UC fields, and front-end loading the cadence with MW observations. Indeed the recovery for MW stars is actually higher than for UC stars in the first two years of the survey. This is a surprising result considering there are only 30 observations per filter allocated for MW fields for the entirety of the LSST survey, while this number is $\sim 70-230$ per filter (depending on filter) for the UC fields. This has been remedied in current versions of the operation simulator, and demonstrates the usefulness of this investigation for optimizing LSST operations.

\subsubsection{Opsim 1_29 North Ecliptic Plane Cadence}

LSST's observation cadence for the SS is optimized for nearby objects rather than for observations of the Galactic halo. They are taken at higher airmass and generally have a lower signal to noise. As shown in the right panel of Figure 12, the successful recovery of an appreciable percentage of RR Lyrae stars' periods is accomplished only after 5 years of the survey. At $\sim 120 \mathrm{kpc}(\langle g\rangle=21.0), \approx 8 \%$ of both RRab and RRc stars have had their periods successfully recovered after 5 years. 
By year 10 this percentage increases to $\approx 44 \%$ at $\sim 120 \mathrm{kpc}$ $(\langle g\rangle=21.0)$ for both RRab and RRc stars. However beyond this distance the recovery of RRc stars falls off precipitously. At a heliocentric distance of $\sim 300 \mathrm{kpc}, \approx 10 \%$ of RRab stars have had their periods recovered while the recovery of RRc stars has fallen to nearly zero. The recovery of RRab stars falls to zero at $\langle g\rangle=24.0$. Note that while this cadence contains a similar number of epochs to the Galactic Plane cadence, they cover a greater spread of phase space and thus, the period recovery is much higher for the North Ecliptic Plane Cadence.

\section{LIGHT CURVE SHAPE RECOVERY}

For each object with a correctly recovered period, we next attempt to recover the input shape of the known light curve. We utilize two methods to accomplish this. First, Fourier-based decompositions of folded light curves have proven to yield useful discriminants between different classes of RR Lyrae stars' variability. A second method includes matching the folded light curve to a discrete set of light curve "templates" that span the space of expected variability shapes (Sesar et al. 2010a). This template-matching technique may yield more accurate light curve shapes than Fourier techniques in cases where there are very few light curve data points, as in the early days of the LSST survey (Kovács \& Kupi 2007). Proper recovery of light curve morphology allows stellar typing and metallicity measurements for RRab stars. We describe each technique and the results of their application in the subsections below.

\subsection{Fourier and Template-matching Techniques}

To explore LSST's ability to recover light curve shapes via Fourier decomposition techniques, the input idealized light curves and the folded light curve realizations generated in our LSST simulation were all fit, via a $\chi^{2}$ minimization, to a nineterm sine Fourier series of the form

$$
m_{i}(t)=A_{0}+\sum_{k=1}^{9} A_{k} \sin \left[2 \pi k f t+\phi_{k}\right],
$$

where $A_{0}$ represents the mean stellar magnitude in filter $i, A_{k}$ is the amplitude of the $k$ th harmonic of the Fourier series, $f=1 / P$ is the frequency (where $P$ is the fitted period of the magnitude variation), and $\phi_{k}$ is the phase of the $k$ th harmonic at $t=0$. This decomposition was performed for all stars that successfully passed our period-fitting criterion set forth in Equation (8). However, we limit our Fourier analysis to data in the $g$ band and the $r$ band, which allow us to estimate metallicities using Equations (2) and (3).

To explore LSST's ability to recover light curve shapes via template-matching techniques, each of Sesar et al.'s (2010a) ugriz RR Lyrae star's templates was fitted to all input light curves, as well as to each period-folded light curve (i.e., light curves with $\delta P<10^{-5}$ ), via a $\chi^{2}$ minimization with the peak-topeak amplitude $A$, mean magnitude $\langle m\rangle$, and phase of maximum brightness $\phi^{\max }$ as the fit variables. The template that returned the smallest value of $\chi^{2}$ per degree of freedom statistic was recorded as the best match to the data. If the template that best described the period-folded data was of the same type as the template that best fit the input light curve, it was considered a successful recovery of the stellar subtype.

We have devised a metric to determine if we successfully recovered the light curve shape and hence a star's metallicity.
Table 6

Template Matching Shape Recovery Mag Limits for a 10 year Survey

\begin{tabular}{lccccc}
\hline \hline Observing & \multicolumn{5}{c}{ Template shape recovery efficiency } \\
Cadence & $97.5 \%$ & $90 \%$ & $50 \%$ & $25 \%$ & $10 \%$ \\
\hline UC & 22.6 & 23.2 & 24.3 & 24.8 & 25.2 \\
DD & 24.0 & 24.6 & 25.4 & 25.7 & 25.9 \\
\hline
\end{tabular}

This technique relies on the $\phi_{31}$ values determined from the direct Fourier decompositions of the data and also from Fourier decompositions of the best-fit templates to the data. To avoid dependency on any particular $\phi_{31}$ versus metallicity measurement (whose precision may improve over time), we use as our core shape recovery metric the value $\Delta \phi_{31}$. This compares the phase of the third harmonic for the input idealized light curve $\phi_{31}^{\text {in }}$ to that derived from either of the two techniques:

$$
\Delta \phi_{31} \equiv \phi_{31}^{\mathrm{fit}}-\phi_{31}^{\text {in }} \text {. }
$$

For each survey mode, we use the bright end of the stellar distribution to determine the empirical scatter of $\Delta \phi_{31}$, which is then compared to the $\Delta \phi_{31}$ distributions for fainter stars. To establish the magnitude range for such "well-measured" light curves, we establish the magnitude bin below which our 10 year period recovery efficiency falls below $90 \%$. We then aggregate the ensemble of $\Delta \phi_{31}$ for all stars in this bin and brighter, for all stars that pass the period-matching criteria. We represent this distribution of bright stars with the variable $\Delta \phi_{31}^{90 ; 10}$; its rms is represented by variable $\sigma_{\Delta \phi 31}$, and should be smaller for the brighter stars and for the longer survey lengths. For all survey lengths, and for all brightnesses, we define the recovery of the correct light curve shape to be when the derived $\phi_{31}^{\text {fit }}$ is within one standard deviation $\left(\sigma_{\Delta \phi 31}\right)$ of the known input value.

Figures 13 and 14 show the histograms of $g$-band $\Delta \phi_{31}$ values for $1,2,5$ and 10 years of data for UC and DD data, respectively. The derived value of the root-mean-square deviation of $\Delta \phi_{31}^{90 ; 10}$ was measured at $\sigma_{\Delta \phi 31}=0.09$ radians for the 10 year DD survey, which is the best representation of the intrinsic $\Delta \phi_{31}$ distribution.

Shown in Figure 15 are the percentages of stars whose $\Delta \phi_{31}$ value was within \pm 0.09 rad for the DD and UC fields, respectively. The dashed lines are derived from Fourier decompositions and the dot-dashed lines are derived from template matching; the solid lines are the period recovery results, which are shown for comparison. Also shown on each plot in the lower panels are the percentage differences from the period recoveries. Two results are immediately apparent in these plots. First, the template matching outperformed the Fourier decompositions at all magnitudes and survey lengths. It is likely that a portion of the Fourier shape recovery inefficiency comes from our implementation of the Minuit2 minimization engine utilized in our fitting routine with 19 Fourier parameters. Fitting millions of light curves leaves us susceptible to infrequent "features" of the minimizer. The template-fitting algorithm utilizes only three parameters and is less susceptible to such effects. The second result is that the shape recovery (particularly for template matching) closely follows the period recovery. The $50 \%$ completeness level for template-matched shape recovery is pushed back only 0.17 mag for 10 years of survey data. A summary of the $g$-band magnitude below which the light curve shape recovery falls to $97.5 \%, 90 \%, 50 \%, 25 \%$, and $10 \%$ as derived from $g$ - and $r$-band data, for UC and DD fields, is given in Tables 6 and 7 for template matching and Fourier decompositions of RR Lyrae stars' light curves, respectively. 

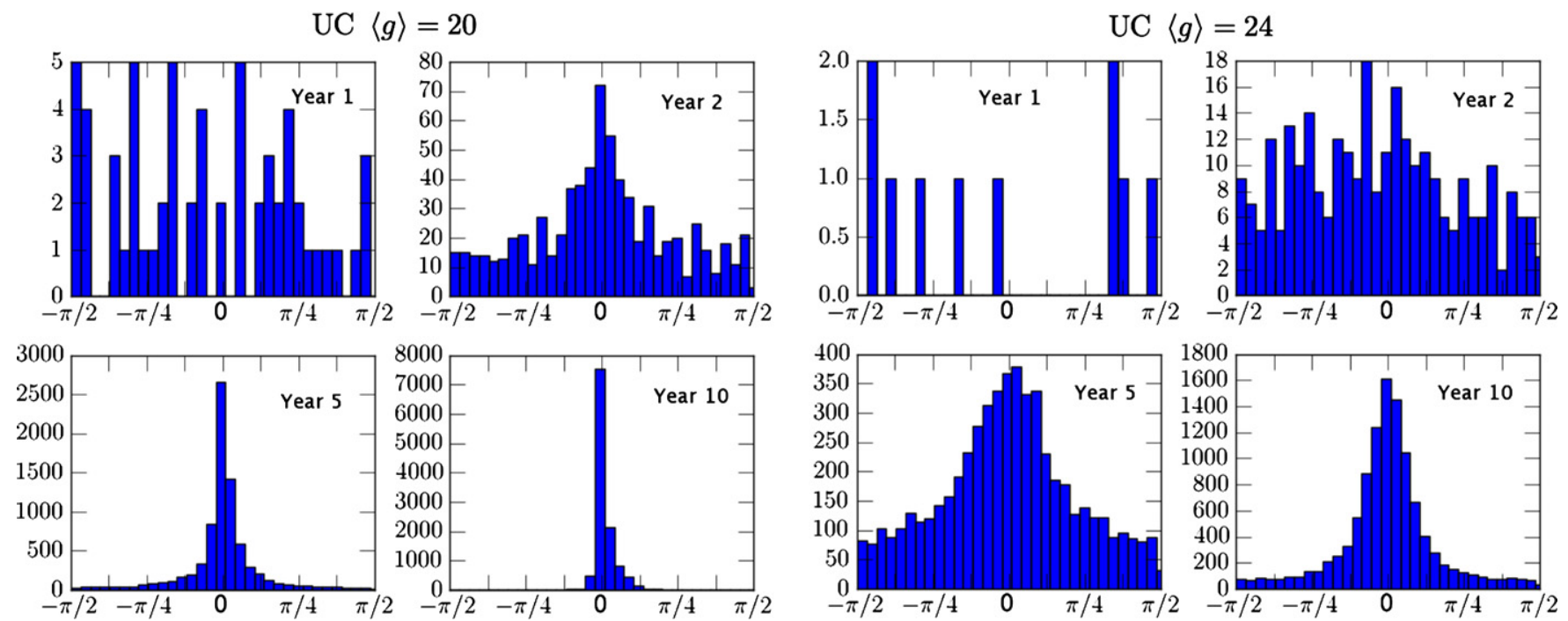

Figure 13. Left: histograms of the Fourier-based $\Delta \phi_{31}$ for $\langle g\rangle=20.0$ RRab stars in UC fields. The root-mean-square values for Years $1,2,5$, and 10 are $0.93,0.65$, 0.36 , and 0.11 , respectively. Right: histograms of the Fourier-based $\Delta \phi_{31}$ for $\langle g\rangle=24.0$ RRab stars in UC fields. The root-mean-square values for Years $1,2,5$, and 10 are $1.14,0.69,0.64$, and 0.42 , respectively.

(A color version of this figure is available in the online journal.)

$$
\mathrm{DD}\langle g\rangle=20
$$
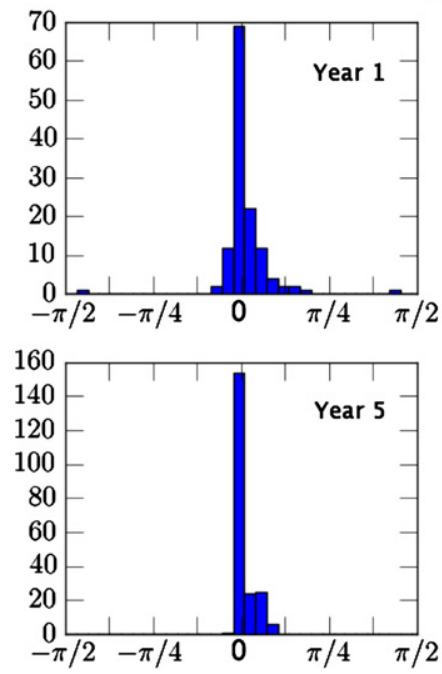


$\mathrm{DD}$
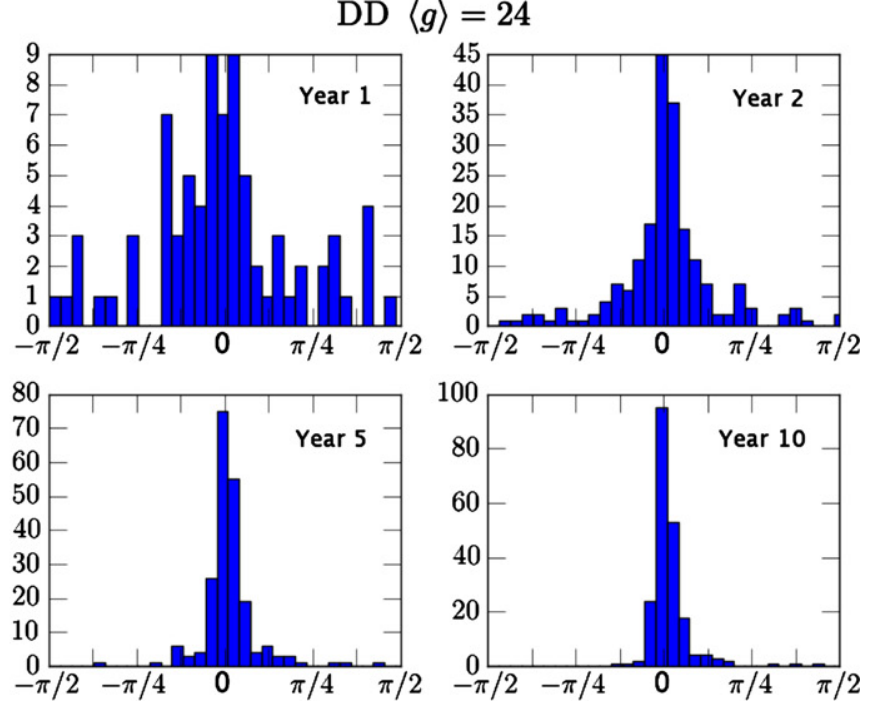

Figure 14. Left: histograms of the Fourier-based $\Delta \phi_{31}$ distribution for $\langle g\rangle=20.0$ RRab stars in DD fields. The root-mean-square values for Years $1,2,5$, and 10 are $0.18,0.08,0.07$, and 0.07 , respectively. Right: histograms of the Fourier-based $\Delta \phi_{31}$ distribution for $\langle g\rangle=24.0$ RRab stars in DD fields. The root-mean-square values for Years $1,2,5$, and 10 are $0.56,0.40,0.20$, and 0.17 , respectively.

(A color version of this figure is available in the online journal.)

Table 7

Fourier Shape Recovery Mag Limits for a 10 year Survey

\begin{tabular}{lccccc}
\hline \hline Observing & \multicolumn{5}{c}{ Fourier shape recovery efficiency } \\
Cadence & $97.5 \%$ & $90 \%$ & $50 \%$ & $25 \%$ & $10 \%$ \\
\hline UC & 20.0 & 21.3 & 23.8 & 24.5 & 24.9 \\
DD & 23.1 & 23.9 & 24.9 & 25.3 & 25.7 \\
\hline
\end{tabular}

\subsection{Photometric Metallicity Measurements}

Figure 16 shows the degradation of the width of the histograms shown in Figures 13 and $14\left(\sigma_{\Delta \phi 31}^{g}\right.$ for $g$-band shape measurements, and $\sigma_{\Delta \phi 31}^{r}$ for the $r$ band) as a function of $g$-band source brightness, for $1,2,5$, and 10 year surveys. The left panel shows this for UC fields, while the right panel shows this for DD fields. We include horizontal lines that reflect an according misfit in $[\mathrm{Fe} / \mathrm{H}]$ that is equivalent to the rms of the current standard for [ $[\mathrm{Fe} / \mathrm{H}]$ versus $\phi_{31}$ estimates, 0.14 dex from Jurcsik \& Kovács (1996). These values are taken from the slopes of Equations (2) and (3): $\Delta[\mathrm{Fe} / \mathrm{H}]^{g}=0.313 \sigma_{\Delta \phi 31}^{g}$ and $\Delta[\mathrm{Fe} / \mathrm{H}]^{r}=0.175 \sigma_{\Delta \phi 31}^{r}$. We caution that the rms about Equations (2) and (3) is $0.26 \mathrm{dex}$, not 0.14 . Thus, these particular relationships are placeholders for improved relationships that are expected by LSST first light.

Assuming that the rms about these relationships may be reduced to approximately $0.14 \mathrm{dex}$, and that the slope on $\phi_{31}$ is similar to as in Equations (2) and (3), the vertical lines in Figure 16 represent the depth to which $[\mathrm{Fe} / \mathrm{H}]$ may be recovered to within the intrinsic scatter of 0.14 dex. For the UC fields, two years of data are not quite sufficient to ensure a measurement of the metallicity, with 5 years sufficient to recover $[\mathrm{Fe} / \mathrm{H}]$ out to $\langle g\rangle=21.5(\sim 150 \mathrm{kpc})$ using $g$-band data, and 

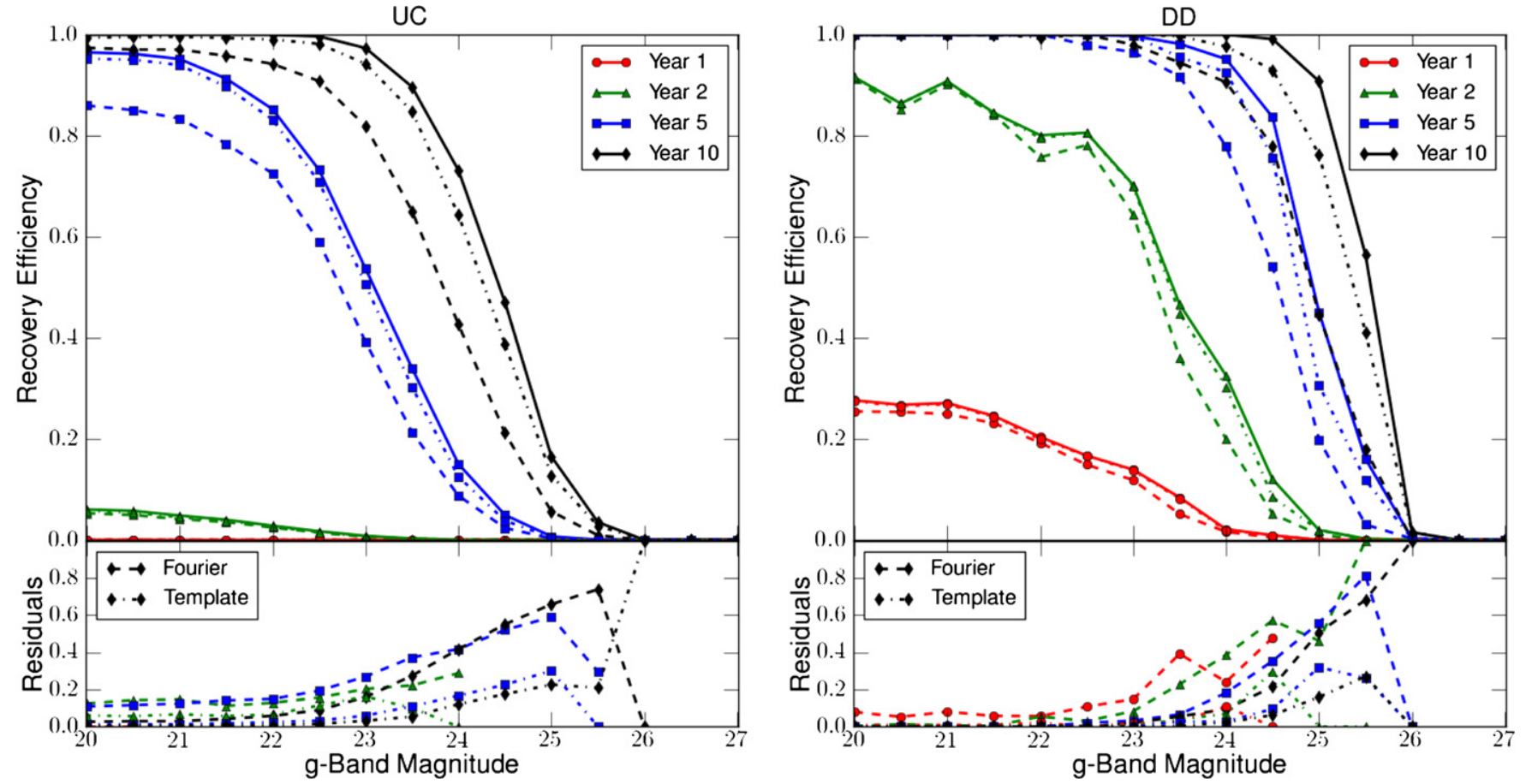

Figure 15. Plots of the Fourier-based shape recovery (dashed) and template-matched shape recovery (dot-dashed) along with the period recovery efficiency (solid), for RRab stars in the UC fields (left) and the DD fields (right). Successful shape recovery is defined as recovering the $\Delta \phi_{31}$ parameter defined in Equation (12) to within one standard deviation of the peak of the 10 year DD $\Delta \phi_{31}$ histogram. In the lower panels we show the residual percentage differences between the period recovery and shape recovery via Fourier-based and template-matching techniques.

(A color version of this figure is available in the online journal.)
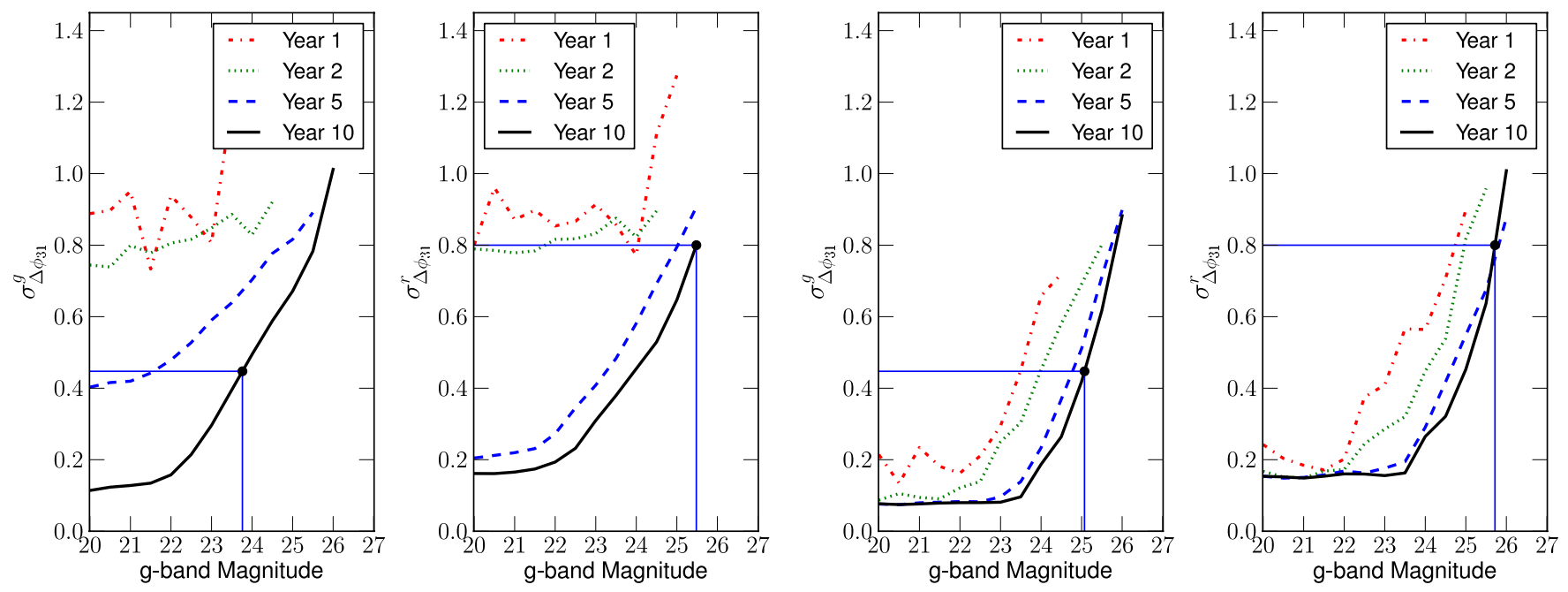

Figure 16. Evolution of $\sigma_{\Delta \phi_{31}}^{g}$ and $\sigma_{\Delta \phi_{31}}^{r}$ with stellar magnitude and survey length. The left panels are for UC fields and the right panels are for DD fields. The horizontal lines reflect a misestimate of $[\mathrm{Fe} / \mathrm{H}]$ by $0.14 \mathrm{dex}$, which is currently the systematic floor for metallicity recovery from light curve shape, assuming the relationships given in Equations (2) and (3). The vertical lines provide the depth to which this is achieved after 10 years of observations. In 10 year survey UC fields, shape recovery is sufficient to recover metallicities to $\langle g\rangle \leqslant 24.0$ using $g$-band light curves, and out to $\langle g\rangle \leqslant 25.5$ using $r$-band light curves. In 10 year survey DD fields, shape measurement is sufficient to recover metallicities to $\langle g\rangle \leqslant 25.0$ using $g$-band light curves, and out to $\langle g\rangle \leqslant 25.5$ using $r$-band light curves.

(A color version of this figure is available in the online journal.)

$\langle g\rangle=25.0(\sim 750 \mathrm{kpc})$ using $r$-band data. Doubling the length of the survey increases the depth of recovery from $g$-band data by $2 \mathrm{mag}$, and $r$-band data by only $0.5 \mathrm{mag}$. In the DD fields, a substantial fraction of the objects will have sufficient shape measurements to recover $[\mathrm{Fe} / \mathrm{H}]$ in the first year of the survey. However, the improvement in $[\mathrm{Fe} / \mathrm{H}]$ recovery between years 5 and 10 is minute. In addition, two years of DD observations equate to 10 years of UC observations, suggesting that, for those objects whose periods are recovered, the DD cadence is far more effective in constraining the shape of the light curves than in estimating the periods initially.

An additional consideration is the improvement in estimates of photometric metallicity that may be obtained by including information from multiple passbands. Metallicity estimates have been shown to be more sensitive to data in bluer passbands, as examined in Ivezić et al. (2008b). This raises the 
Table 8

Magnitude Limits for Subtype Recovery from a 10 year LSST Survey

\begin{tabular}{lccccc}
\hline \hline Observing & \multicolumn{5}{c}{ Subtype recovery efficiency } \\
Mode & $97.5 \%$ & $90 \%$ & $50 \%$ & $25 \%$ & $10 \%$ \\
\hline UC & 23.0 & 23.5 & 24.4 & 24.9 & 25.3 \\
DD & 24.6 & 25.0 & 25.6 & 25.8 & 25.9 \\
OL & 23.1 & 23.7 & 24.8 & 25.2 & 25.5 \\
MW & $\ldots$ & $\ldots$ & $\ldots$ & $\ldots$ & 21.4 \\
SS & $\ldots$ & $\ldots$ & $\ldots$ & 22.2 & 23.0 \\
\hline
\end{tabular}

possibility of using $u$-band light curve shapes to additionally constrain $[\mathrm{Fe} / \mathrm{H}]$, if sufficient relationships are derived from extant data. Using three passbands of information simultaneously could result in greater than $40 \%$ error improvement for photometrically determined metallicities as compared to using a single passband of information.

\subsection{Type Recovery Results}

Here we quantify LSST's ability to recovery the input RR Lyrae stars' stellar subtypes, RRab or RRc. This was done by phasing all the light curves with the best-fit period as determined in Section 4.1. The best-fit templates were then determined as described in Section 5.1. Finally, we determined the fraction of all stars for which the best-fit template subtype ( $a b$ or $c$ ) matched the input light curves subtype. Table 8 lists the efficiencies with which the RR Lyrae star subtypes were recovered.

\section{BRIGHTNESS RECOVERY}

Mean magnitudes of variable stars are typically defined by either a simple mean in magnitude, or by a star's flux-averaged magnitude (FAM; Jameson 1986). The latter is obtained by converting the light curve from magnitude units to flux units and then integrating the light curve. The FAM is essentially the flux the star would have if it were not pulsating and relates the star's emergent intensity to the energy generated by the core nuclear reactions, which are not affected by the star's pulsation and are of interest to stellar astrophysicists.

For stars near the detection limit of a survey, observations taken when the star is at peak brightness are more likely to yield detections than when the star is faint, biasing mean magnitudes toward brighter magnitudes. We utilize a method to derive FAMs that do not suffer this bias as strongly. In our analysis the FAM is calculated by first identifying the best-fit template to the data and then integrating this best-fit template, which assumes known RR Lyrae stars' light curve shape, rather than by deriving the FAM directly from the data itself. For a star near the detection limit, the shape of the light curve near peak brightness will provide a useful discriminant between templates if a sufficient phase range is present in the data.

To assess brightness recovery, the FAMs calculated from the best-fit templates are compared to the FAMs calculated from the input light curves to determine the errors on each recovered FAM. The weighted statistical means of the FAM errors $\bar{\epsilon}_{f}(m)$ ( $f=g, r, i)$ in each magnitude bin and subsurvey duration were calculated as shown below in Equation (13):

$$
\bar{\epsilon}_{f}(m)=\frac{1}{N_{s}} \sum_{i=1}^{N_{s}} w_{i} \bar{\epsilon}_{i}(m) .
$$

Here, $w_{i}$ are the weights of the individual stars as described in Equation (9), $\bar{\epsilon}_{i}(m)$ is the mean FAM error for star $i$ at magnitude
Table 9

Flux Recovery Limits $\left(\bar{\epsilon}_{f}(m) \leqslant 0.03\right)$ from a 10 year LSST Survey

\begin{tabular}{lccc}
\hline \hline Cadence & \multicolumn{3}{c}{ Passband } \\
& $g$ & $r$ & $i$ \\
\hline DD & 24.2 & 24.1 & 23.8 \\
UC & 24.7 & 24.5 & 24.1 \\
\hline
\end{tabular}

$m$ averaged across all fields for a given cadence, and $N_{s}$ is the total number of stars. The weighted statistical means of the FAM errors $\bar{\epsilon}_{k}(m)$ as well as the rms deviation about this mean $\sigma_{\bar{\epsilon}}(m)$ were recorded.

Table 9 lists the magnitudes where $\bar{\epsilon}_{f}(m)$ exceeds the 0.03 mag systematic error in the RR Lyrae stars' brightness calibration for the DD, UC, and MW fields. The results do not show much variation with survey length, indicating that magnitudes are, for the most part, recovered in the first year of the survey for both DD and UC fields. The rms deviation of the $\bar{\epsilon}_{f}(m)$ distributions increases with the $g$-band magnitude, indicating that the mean brightnesses are overestimated as the stars become dimmer and the light curve points near brightness minimum are recovered less efficiently than the points near brightness maximum thus reducing the efficiency of successful template matching.

\section{DISCUSSION}

We have undertaken an extensive simulation to assess LSST's capability to characterize RR Lyrae stars throughout the course of its 10 year survey. This simulation includes realistic temporal sampling of field centers using five specific observing modes as implemented in operation simulation version opsim1_29, and realistic photometric measurements that include simulated observing conditions for each individual epoch. Within this framework we have evaluated the light curves of $\sim 36$ million simulated RR Lyrae stars' light curves. The input light curve shapes sample the known RRab and RRc population, and include the known color evolution of these objects within a pulsational period (with the exception of $z-y$ color, which is set to 0.0 here). Our 40 input light curve shapes are evaluated in 6 of the LSST filters, each being placed at 1007 field centers that sample 5 specific observing modes. We evaluate each of these light curves in $15 \mathrm{mag}$ bins to assess the degradation of the photometry with apparent magnitude, and in subsurveys from 1 to 10 years in length. These subsurveys illustrate the science achievable as a function of the survey length and its limits as well as informing the question of "what is the appropriate survey length for the LSST survey to achieve its science goals for RR Lyrae stars as tools for measuring Galactic structure?."

Our investigation of period recovery shows that the majority of Galactic halo RR Lyrae periods will be recovered in 2 years of DD observations, and in 5 years of UC observations. To recover the periods of RR Lyrae at $\sim \mathrm{Mpc}$ distances requires a minimum of 5 years of DD observations. A slight modification of the DD cadence, incorporating a field revisit within a night, may decrease the amount of time it takes to recover the periods of such short-period objects. If the saturation of science occurs more rapidly, the DD field centers may be moved after a couple of years to accomplish similar science at a different pointing. This is an idea under active consideration by LSST. The overlap (OL) of UC fields show an increased recovery of periods compared to UC fields, as expected. However, actual 
observations by LSST may include dithering of UC pointings, making the contrast between UC and OL regions less stark than presented here. Finally, observations in the North Ecliptic of the SS and that are near the MW Galactic plane are expected to return marginal efficiency on period recovery, given the small number of total observations planned for these cadences and the phase clustering of the samples. In this study, we identified a flaw in the implementation of the MW observations, which has since been remedied and will help distribute the MW observations equally over the 10 years of LSST. This will enable long-term studies of proper motion and parallax in the Galactic plane, and demonstrates the effectiveness of early-term studies such as this.

We have also assessed the ability to recover shapes of the period-folded light curves, using the Fourier term $\phi_{31}$ as our metric. This has been shown to be a useful estimate of $[\mathrm{Fe} / \mathrm{H}]$ metallicity in the $V$ band by Jurcsik \& Kovács (1996), and more recently in the $g$ band by Sesar et al. (2010a). We present an $r$-band relationship here based on the same multiband data used by Sesar et al. (2010a) that shows a shallower slope on $\phi_{31}$ than in the $g$ band, and is thus a less sensitive indicator. We demonstrate that, using these extant $g$ - and $r$-band relationships, we may recover metallicities of objects through their shapes down to an assumed rms of 0.14 dex with a loss in efficiency of only a few percent compared to period recovery. We use the technique of template matching to calculate FAMs, recover the shapes of period-folded light curves, and to discriminate between RRab and RRc subtypes. We demonstrate that the template-matching approach will be particularly effective at recovering the brightnesses, light curve shapes, and RR Lyrae stars' subtypes very early in the survey.

One concern for a simulation of this sort is that the process of mining the RR Lyrae stars from the data was not simulated. Sesar et al. (2010a) have shown that RR Lyrae stars can be efficiently mined from survey data via a blind search by making standard cuts on color, variability, period, and amplitude to identify a set of candidate RR Lyrae stars. They further refined their sample using the rms scatter $\sigma_{g}$ and $\sigma_{r}$ in the $g$ - and $r$ band light curves, respectively, since they found that $97 \%$ of RR Lyrae stars have $\left|\sigma_{g}-1.42 \sigma_{r}\right|<0.03$. Using these cuts was sufficient to identify RRab stars, however the population of RRc candidates would still very likely be contaminated by eclipsing binaries with similar light curves. Comparison of the rms scatter $\sigma_{g-r}$ in the $g-r$ light curves with the $\sqrt{\sigma_{g}^{2}+\sigma_{r}^{2}}$, which is sensitive to the covariance between the $g$ and $r$ bands, allows efficient elimination of this contamination. We anticipate that the use of these cuts in combination with template matching should yield a contamination free sample or RR Lyrae stars from LSST data for which analysis would then proceed similarly to our study here.

\section{CONCLUSIONS}

We have evaluated the limits of the RR Lyrae star science that may be expected from LSST data in the context of performing galactic archeology in the Milky Way halo and in the Local Group of galaxies. The results shown here indicate that the LSST will possess unprecedented capabilities for measuring the Milky Way's history of Galactic mergers by mapping the spatial distributions (with $\sim 5 \%$ uncertainty in distance) and metallicity of halo stellar overdensities and field stars (with a $\sim 0.1-0.2 \mathrm{dex}$ precision), using RR Lyrae stars discovered across $3 \pi$ sr, and out to $\sim 600 \mathrm{kpc}$ for $50 \%$ of RRab stars in UC fields and out to $\sim 250 \mathrm{kpc}$ for $50 \%$ of RRc stars after a 10 year survey.
With RR Lyrae stars detected to these distances, LSST will be able to search for halo streams and dwarf satellite galaxies over half of the Local Group (Ivezić et al. 2008a). In addition to detection of these structures, LSST will also be able to estimate their metallicity using a relation between the light curve shape and metallicity of RR Lyrae stars. By mapping the number density and metallicity of stars in the Local Group, LSST will more strongly constrain the formation history of the Local Group and the galaxy formation process in general (for more details, see Section 7 in the LSST Science Book).

The sheer number of exquisitely sampled RR Lyrae stars' light curves will provide new insight into the astrophysics of these important standard candles. For example, our empirical knowledge of RR Lyrae stars' period distributions and trends in absolute magnitude with period, age, and metallicity comes from studying properties of RR Lyrae stars in globular clusters, where the distance determinations may not be precise enough (at the 20\% level). The range of distances within the Large Magellanic Cloud (LMC) is smaller than the uncertainty in relative distances between globular clusters in our Galaxy, and LSST is expected to find about $10^{5}$ RR Lyrae stars over the face of LMC (LSST Science Collaborations 2009, LSST Science Book Section 6.2.1). Ages and metallicities of the oldest stars (the parent population of the RR Lyrae stars) in any given location in the Clouds may be gleaned from an analysis of the local color-magnitude diagrams and trends in RR Lyrae stars' properties with parent population may be directly mapped for the first time (see LSST Science Book Section 6.2 for more details on the analysis of LMC color-magnitude diagrams).

One exciting possibility, and a strong driver for precisely measured light curves, would be a discovery of an RR Lyrae star in an eclipsing binary system. Spectroscopic observations of such a system would provide us with an estimate of the dynamical mass of the RR Lyrae star, allowing for a direct comparison with masses determined from pulsation and evolutionary models. A Classical Cepheid in an eclipsing binary system has already been found (Pietrzyński et al. 2010), but an RR Lyrae star in an eclipsing binary system is yet to be discovered.

While LSST may discover RR Lyrae stars beyond $\sim 600 \mathrm{kpc}$ in UC fields, the periods, metallicities, and flux-averaged magnitudes of these stars will be more uncertain, as Figures 9, 15, and 16 show. Whether the detected RR Lyrae stars will still be useful even with these uncertainties will vary from study to study. Nonetheless, measurements of times of maximum and minimum and recovery of periods to high precision at these distances will facilitate spectroscopic analyses with excellent control of systematic complications such as color and temperature changes over the pulsation cycle, and complex shocks that occur in photospheres of RR Lyrae stars at maximum light. Moreover, the long time-baseline, high-cadence photometry of such large numbers of RR Lyrae stars should allow models of the fundamental causes of Blazkho variability to be tested with excellent statistics.

This work was supported by the National Science Foundation under Scientific Program Order No. 9 (AST-0551161) through Cooperative Agreement AST-0132798. We also thank the Office of Educational Programs at Brookhaven National Laboratory with LSST Educational Outreach Programs for their support. We used the "Athena" compute cluster at the University of Washington for much of the light curve phasing and Fourier decomposition. The authors thank the referee for very insightful comments that have improved the quality of the paper. 


\section{REFERENCES}

Adelman-McCarthy, J. K., Agüeros, M. A., Allam, S. S., et al. 2008, ApJS, 175, 297

Alves-Brito, A., Forbes, D. A., Mendel, J. T., Hau, G. K. T., \& Murphy, M. T. 2009, MNRAS, 395, L34

Aoki, W., Arimoto, N., Sadakane, K., et al. 2009, A\&A, 502, 569

Bailey, S. I. 1902, Ann. Harvard College Obs., 38, 1

Bell, E. F., Zucker, D. B., Belokurov, V., et al. 2008, ApJ, 680, 295

Belokurov, V., Evans, N. W., Bell, E. F., et al. 2007a, ApJ, 657, L89

Belokurov, V., Evans, N. W., Irwin, M. J., Hewett, P. C., \& Wilkinson, M. I. 2006a, ApJ, 637, L29

Belokurov, V., Evans, N. W., Irwin, M. J., et al. 2007b, ApJ, 658, 337

Belokurov, V., Walker, M. G., Evans, N. W., et al. 2008, ApJ, 686, L83

Belokurov, V., Walker, M. G., Evans, N. W., et al. 2009, MNRAS, 397, 1748

Belokurov, V., Walker, M. G., Evans, N. W., et al. 2010, ApJ, 712, L103

Belokurov, V., Zucker, D. B., Evans, N. W., et al. 2006b, ApJ, 642, L137

Belokurov, V., Zucker, D. B., Evans, N. W., et al. 2006c, ApJ, 647, L111

Belokurov, V., Zucker, D. B., Evans, N. W., et al. 2007c, ApJ, 654, 897

Brook, C. B., Kawata, D., Gibson, B. K., \& Flynn, C. 2004, MNRAS, 349, 52

Bullock, J. S., \& Johnston, K. V. 2005, ApJ, 635, 931

Cáceres, C., \& Catelan, M. 2008, ApJS, 179, 242

Chaboyer, B. 1999, in Post-Hipparcos Cosmic Candles, ed. A. Heck \& F. Caputo (Astrophysics and Space Science Library, Vol. 237; Dordrecht: Kluwer), 111 Collinge, M. J., Sumi, T., \& Fabrycky, D. 2006, ApJ, 651, 197

Cook, K. H., Saha, A., Pierfedrici, F., et al. 2004, BAAS, 36, 1528

Cooper, A. P., Cole, S., Frenk, C. S., et al. 2010, MNRAS, 406, 744

Deason, A. J., Belokurov, V., \& Evans, N. W. 2011, MNRAS, 416, 2903

De Lucia, G., \& Helmi, A. 2008, MNRAS, 391, 14

Dolphin, A. E. 2002a, MNRAS, 332, 91

Dolphin, A. E. 2002b, in ASP Conf. Ser. 274, Observed HR Diagrams and Stellar Evolution, ed. T. Lejeune \& J. Fernandes (San Francisco, CA: ASP), 450

Dolphin, A. E., Makarova, L., Karachentsev, I. D., et al. 2001, MNRAS, 324, 249

Dolphin, A. E., Saha, A., Olszewski, E. W., et al. 2004, AJ, 127, 875

Durrell, P. R., Harris, W. E., \& Pritchet, C. J. 2001, AJ, 121, 2557

Font, A. S., Johnston, K. V., Ferguson, A. M. N., et al. 2008, ApJ, 673, 215

Frebel, A., Johnson, J. L., \& Bromm, V. 2008, in IAU Symp. 255, Low-

Metallicity Star Formation: From the First Stars to Dwarf Galaxies, ed. L. K.

Hunt, S. Madden, \& R. Schneider (Cambridge: Cambridge Univ. Press), 336

Fukugita, M., Ichikawa, T., Gunn, J. E., et al. 1996, AJ, 111, 1748

Grillmair, C. J. 2006a, ApJ, 645, L37

Grillmair, C. J. 2006b, ApJ, 651, L29

Grillmair, C. J. 2009, ApJ, 693, 1118

Grillmair, C. J., \& Dionatos, O. 2006a, ApJ, 641, L37

Grillmair, C. J., \& Dionatos, O. 2006b, ApJ, 643, L17

Grillmair, C. J., \& Johnson, R. 2006, ApJ, 639, L17

Helmi, A., \& White, S. D. M. 1999, MNRAS, 307, 495

Helmi, A., White, S. D. M., de Zeeuw, P. T., \& Zhao, H. 1999, Nature, 402, 53

Ibata, R., Chapman, S., Ferguson, A. M. N., et al. 2004, MNRAS, 351, 117

Ibata, R., Chapman, S., Ferguson, A. M. N., et al. 2005, ApJ, 634, 287

Ibata, R., Irwin, M., Lewis, G., Ferguson, A. M. N., \& Tanvir, N. 2001a, Nature, 412, 49

Ibata, R., Irwin, M., Lewis, G. F., \& Stolte, A. 2001b, ApJ, 547, L133

Ibata, R., Martin, N. F., Irwin, M., et al. 2007, ApJ, 671, 1591

Ibata, R. A., Gilmore, G., \& Irwin, M. J. 1994, Nature, 370, 194

Ibata, R. A., Gilmore, G., \& Irwin, M. J. 1995, MNRAS, 277, 781

Ibata, R. A., Lewis, G. F., Irwin, M. J., \& Cambrésy, L. 2002, MNRAS, 332, 921

Ivezić, Ž., Tyson, J. A., Allsman, R., et al. 2008a, arXiv:0805.2366

Ivezić, Ž., Vivas, A. K., Lupton, R. H., \& Zinn, R. 2005, AJ, 129, 1096

Ivezić, Ž., Goldston, J., Finlator, K., et al. 2000, AJ, 120, 963

Ivezić, Ž., Sesar, B., Jurić, M., et al. 2008b, ApJ, 684, 287
Jameson, R. F. 1986, Vistas Astron., 29, 17

Johnston, K. V., Bullock, J. S., Sharma, S., et al. 2008, ApJ, 689, 936

Jurcsik, J., \& Kovács, G. 1996, A\&A, 312, 111

Kalirai, J. S., Gilbert, K. M., Guhathakurta, P., et al. 2006, ApJ, 648, 389

Keller, S. C., Murphy, S., Prior, S., Da Costa, G., \& Schmidt, B. 2008, ApJ, 678, 851

Kinemuchi, K. 2004, PhD thesis, Michigan State Univ., MI

Kinemuchi, K., Smith, H. A., Woźniak, P. R., \& McKay, T. A. 2006, AJ, 132, 1202

Kirby, E. N., Guhathakurta, P., Bullock, J. S., et al. 2009, arXiv:0902.2591

Kleyna, J., Wilkinson, M. I., Evans, N. W., Gilmore, G., \& Frayn, C. 2002, VizieR Online Data Catalog, 733, 792

Kleyna, J. T., Wilkinson, M. I., Evans, N. W., \& Gilmore, G. 2001, ApJ, 563, L115

Kleyna, J. T., Wilkinson, M. I., Evans, N. W., \& Gilmore, G. 2004, MNRAS, 354, L66

Kleyna, J. T., Wilkinson, M. I., Gilmore, G., \& Evans, N. W. 2003, ApJ, 588, L21

Koch, A., \& Côté, P. 2010, A\&A, 517

Koch, A., Côté, P., \& McWilliam, A. 2009, A\&A, 506, 729

Koch, A., \& Rich, R. M. 2010, AJ, 139, 2279

Kollmeier, J. A., Gould, A., Shectman, S., et al. 2009, ApJ, 705, L158

Kovács, G., \& Kupi, G. 2007, A\&A, 462, 1007

LSST Science Collaborations. 2009, arXiv:0912.0201

Lupton, R. H., Gunn, J. E., \& Szalay, A. S. 1999, AJ, 118, 1406

Mackey, A. D., Huxor, A. P., Ferguson, A. M. N., et al. 2010a, ApJ, 717, L11

Mackey, D., Huxor, A., Ferguson, A., et al. 2010b, arXiv:1005.3812

Majewski, S. R., Beaton, R. L., Patterson, R. J., et al. 2007, ApJ, 670, L9

Majewski, S. R., Ostheimer, J. C., Rocha-Pinto, H. J., et al. 2004, ApJ, 615, 738

Marconi, M., Cignoni, M., Di Criscienzo, M., et al. 2006, MNRAS, 371, 1503

Martin, N. F., Ibata, R. A., Bellazzini, M., et al. 2004, MNRAS, 348, 12

Morgan, S. M., Wahl, J. N., \& Wieckhorst, R. M. 2007, MNRAS, 374, 1421

Mouhcine, M., Rich, R. M., Ferguson, H. C., Brown, T. M., \& Smith, T. E. 2005, ApJ, 633, 828

Newberg, H. J., Yanny, B., Grebel, E. K., et al. 2003, ApJ, 596, L191

Newberg, H. J., Yanny, B., Rockosi, C., et al. 2002, ApJ, 569, 245

Newberg, H. J., Yanny, B., \& Willett, B. A. 2009, ApJ, 700, L61

Odenkirchen, M., Grebel, E. K., Dehnen, W., et al. 2003, AJ, 126, 2385

Pejcha, O., \& Stanek, K. Z. 2009, ApJ, 704, 1730

Pietrzyński, G., Thompson, I. B., Gieren, W., et al. 2010, Nature, 468, 542

Prantzos, N. 2008, arXiv:0709.0833

Read, J. I., Lake, G., Agertz, O., \& Debattista, V. P. 2008, MNRAS, 389, 1041

Reimann, J. D. 1994, PhD thesis, Univ. California

Richardson, J. C., Ferguson, A. M. N., Mackey, A. D., et al. 2009, MNRAS, 396, 1842

Robertson, B., Bullock, J. S., Font, A. S., Johnston, K. V., \& Hernquist, L. 2005, ApJ, 632, 872

Rocha-Pinto, H. J., Majewski, S. R., Skrutskie, M. F., Crane, J. D., \& Patterson, R. J. 2004, ApJ, 615, 732

Salaris, M., \& Cassisi, S. (ed.) 2005, Evolution of Stars and Stellar Populations (Chichester: Wiley-VCH)

Searle, L., \& Zinn, R. 1978, ApJ, 225, 357

Seljak, U., Slosar, A., \& McDonald, P. 2006, J. Cosmol. Astropart. Phys., JCAP10(2006)014

Sesar, B., Ivezić, Ž., Grammer, S. H., et al. 2010a, ApJ, 708, 717

Sesar, B., Vivas, A. K., Duffau, S., \& Ivezić, Ž. 2010b, ApJ, 717, 133

Sesar, B., Ivezić, Ž, Lupton, R. H., et al. 2007, AJ, 134, 2236

Spergel, D. N., Bean, R., Doré, O., et al. 2007, ApJS, 170, 377

Szczygieł, D. M., Pojmański, G., \& Pilecki, B. 2009, Acta Astron., 59, 137

Tanaka, M., Chiba, M., Komiyama, Y., et al. 2010, ApJ, 708, 1168

Vivas, A. K., \& Zinn, R. 2006, AJ, 132, 714

Watkins, L. L., Evans, N. W., Belokurov, V., et al. 2009, MNRAS, 398, 1757

Wils, P., Lloyd, C., \& Bernhard, K. 2006, MNRAS, 368, 1757

Yanny, B., Newberg, H. J., Grebel, E. K., et al. 2003, ApJ, 588, 824 